\title{
Compilation of small ribosomal subunit RNA structures
}

\author{
Jean-Marc Neefs, Yves Van de Peer, Peter De Rijk, Sabine Chapelle and Rupert De Wachter* \\ Departement Biochemie, Universiteit Antwerpen (UIA), Universiteitsplein 1, B-2610 Antwerp, Belgium
}

\begin{abstract}
The database on small ribosomal subunit RNA structure contained 1804 nucleotide sequences on April 23, 1993. This number comprises 365 eukaryotic, 65 archaeal, 1260 bacterial, 30 plastidial, and 84 mitochondrial sequences. These are stored in the form of an alignment in order to facilitate the use of the database as input for comparative studies on higherorder structure and for reconstruction of phylogenetic trees. The elements of the postulated secondary structure for each molecule are indicated by special symbols. The database is available on-line directly from the authors by ftp and can also be obtained from the EMBL nucleotide sequence library by electronic mail, ftp, and on CD ROM disk.
\end{abstract}

\section{CONTENTS OF THE DATABASE}

The database on small ribosomal subunit RNA (further abbreviated as SSU rRNA) currently contains 1804 nucleotide sequences, stored in the form of an alignment and containing the postulated secondary structure pattern in encoded form. This number comprises 365 eukaryotic cytoplasmic, 65 archaeal, 1260 bacterial, 30 plastidial, and 84 mitochondrial SSU rRNAs. Partial sequences are included if the combined length of the sequenced segments corresponds to homologous segments in Escherichia coli SSU rRNA amounting to at least $70 \%$ of the chain length of the latter molecule.

Previous compilations $(1-5)$ included a table listing for each entry the species name, further specifications such as the strain, variety, or tissue used for isolation of the gene, taxonomic position of the species, length and completeness of the sequence, and accession number in nucleotide sequence libraries. Complete literature references were also included for each entry. Because the number of available structures has nearly doubled since publication of the preceding compilation (5), such a table and the accompanying references would now require an estimated 20 pages just to cover the additional structures entered in the database during the last year. Table 1 has therefore been restricted to a list of the names of species for which the SSU rRNA structure is recorded in the database. However, this list covers all the structures now filed, not just those added since the preceding compilation. Instructions for obtaining the complete table including further specifications and literature references, separately or together with the structural data, are given below.

\section{TAXONOMIC CLASSIFICATION OF SPECIES}

For the Eukarya, the taxonomic classification of the species listed in Table 1 is according to Brusca and Brusca (6) for the Animalia, according to Cronquist (7) for the higher plants, according to Ainsworth et al. (8) for the higher fungi, and according to Corliss (9) for the remaining eukaryotes.

For the Bacteria and the Archaea, the classification followed is based on the phylogeny described by Woese and coworkers $(10-12)$. However, the assignment of a species to one of the taxa distinguished by these authors is often problematic. To our knowledge there does not exist a list assigning each bacterial or archaeal species to one of the divisions or subdivisions that they distinguish. Moreover, many sequences now become available through deposition in one of the nucleotide sequence libraries, which in many cases is not (yet) accompanied by publication in a journal. The bacterial and archaeal SSU rRNA sequences deposited in these libraries are accompanied by a taxonomic description which does not correspond to that of Woese et al. $(10-12)$ but is based on Bergey's Manual of Systematic Bacteriology (13). Even the sequences described in the literature are not always accompanied by an assignment of the species to one of the taxa distinguished by Woese and coworkers. In order to obtain a more or less consistent classification, we have therefore constructed an evolutionary tree from the alignment of all archaeal, bacterial, and plastidial SSU rRNA sequences, 1355 in total. The method followed for constructing the tree has been described in detail elsewhere (14). In short, a dissimilarity matrix was computed, corrected for multiple mutation (15), and a tree derived by neighbour-joining (16). The outline of the resulting tree is shown in Fig. 1. Most of the clusters visible in the tree correspond to the archaeal and bacterial divisions and subdivisions distinguished by Woese and coworkers (10-12). However, in the bacterial subtree, the genera Fibrobacter and Fusobacterium do not integrate in any of the clusters. They are therefore listed separately in Table 1.

It should be noted that the evolutionary distances between the branching points leading to the clusters visible in the tree of Fig. 1 are very small, especially for the major clusters of the Bacteria. Trees such as this one were constructed periodically as the SSU rRNA sequence alignment grew in size. Although the clusters indicated in Fig. 1 were reproducibly formed, the branching order of these clusters was not constant but changed as a function of the composition of the sequence collection. However, the tree can serve to assign bacterial species to a given division or subdivision because, although the relative position of the clusters

* To whom correspondence should be addressed 
is variable, the appurtenance of each bacterial species to a particular cluster is stable.

In Table 1, for the Bacteria, no hierarchical distinction is made between divisions (e.g. the spirochetes) and subdivisions (e.g. the $\alpha, \beta, \gamma, \delta$, and $\epsilon$ subdivisions of the division Proteobacteria). This is because the subdivisions do not always form together a monophyletic cluster. As an example, in the tree of Fig. 1, the Proteobacteria $\epsilon$ group is separated from the monophyletic cluster formed by the Proteobacteria $\alpha, \beta, \gamma$ and $\delta$ groups. As for the division of Gram positive bacteria and relatives, its two subdivisions of high and low GC contents almost never form together a monophyletic cluster in the trees that we obtain. For the Archaea, on the contrary, a distinction is made between the divisions Crenarchaeota and Euryarchaeota (12). The latter division is subdivided into 8 subdivisions. Of these, the Methanobacteriales, Methanococcales, Thermococcales and Methanopyrales correspond to lineages distinguished by Olsen and Woese (12). The Methanomicrobiales group of the latter authors comprises the Methanomicrobium group, the Halobacteria, and Archaeoglobus fulgidus in the tree of Fig. 1.

\section{SECONDARY STRUCTURE MODEL}

Prokaryotic and eukaryotic models, nucleotide variability

Fig. 2 shows the prokaryotic secondary structure model, applicable to SSU rRNAs from archaea, bacteria, plastids, and mitochondria. The model of Fig. 3 applies to eukaryotic cytoplasmic SSU rRNAs. In contrast to the corresponding figures in the preceding compilation (5), the models shown in Fig. 2 and 3 do not simply distinguish between conserved and variable areas, but give a more detailed description of the variability of each site. The latter is defined as the ratio of the substitution rate at the considered site to the average substitution rate for the entire molecule. The quantitative derivation of the variability of each site from the sequence alignment is described in detail elsewhere (17). Sites that are absolutely conserved, and those that are occupied only in a limited number of SSU rRNAs, are indicated by special symbols. The remaining ones were partitioned into five equally large categories of increasing variability. In Fig. 2 and 3, such sites are represented by dots with a diameter commensurate with their variability. Variable areas previously distinguished on a more intuitive basis and indicated on the general secondary structure models of the preceding compilation (5) are still shown on Figg. 2 and 3 as V1 to V9.

\section{Helix numbering system and changes made to the models}

Helices are given a different number if separated by a multibranched loop (e.g. helices 9 and 10), by a pseudoknot loop (e.g. helices 1 and 2), or by a single stranded area that does not form a loop (e.g. helices 2 and 32). A single number is attributed to 50 'universal' helices, which are present in all hitherto known SSU rRNAs from Archaea, Bacteria and plastids. They are also present in all known eukaryotic SSU rRNAs except in those of Microsporidia, where some of these helices are missing. The number of universal helices has risen from $\mathbf{4 8}$ in the preceding compilation (5) to 50 because the tertiary interaction described by Woese and Gutell (18) has been taken into account. This interaction effectively transforms the helix previously numbered 19 into three helices now numbered 19 to 21 .

Helices specific to the prokaryotic model (Fig. 2) are given composite numbers of the form Pa-b, where $a$ is the number of the preceding universal helix and $b$ sequentially numbers all helices inserted between universal helices a and $a+1$. Helices specific to the eukaryotic model (Fig. 3) are similarly numbered Ea-b. In Figg. 2 and 3, not all eukaryote- and prokaryotespecific helices that are encountered in various species are indicated, because these models in fact have the shape of the Escherichia coli and Saccharomyces cerevisiae SSU rRNA secondary structure models, respectively. As an example, in Drosophila melanogaster SSU rRNA, the loop separating helices E23-2 and E23-5 is a multifurcation bearing two more helices numbered E23-3 and E23-4 (5). These and other supernumerary helices that are present in a minority of SSU rRNAs are not indicated in Figg. 2 and 3, one of the reasons being that the variability of the sites composing such helices cannot be computed in a dependable manner. However, in Table 2 the presence or absence of eukaryote specific helices in SSU rRNAs of different taxa is summarized.

Mitochondrial SSU rRNAs, though they can be described by the prokaryotic model, show extreme variability in length, ranging from about 600 nucleotides in flagellates to about 2000 nucleotides in plants. This coincides with the absence of several universal helices in the smaller molecules and with the presence of extra helices of the P-series in the larger ones. A tentative helix occupancy table for mitochondrial SSU rRNAs and examples of secondary structure models can be found in a previous compilation (4). The alignment of, and transposition of secondary structure models to, mitochondrial SSU rRNAs is less dependable than for other SSU rRNAs, not only because of the variability in length, but also because some of the mitochondrial sequences are very monotonous due to their high AU contents.

\section{Examples of secondary structure models}

Figg. 4 to 7 are examples of secondary structure models applied to specific SSU rRNA sequences. Fig. 4 represents the SSU rRNA of the bacterium Escherichia coli, whereas the SSU rRNA of the halophilic archaebacterium Halobacterium halobium is shown in Fig. 5. The eukarya are represented by the structure of SSU rRNAs of the red alga Palmaria palmata in Fig. 6, and of the polymastigote Giardia duodenalis in Fig. 7. The latter model is shown as an example of a molecule possessing a restricted number of helices in variable areas V2 (helices 9 to 11) and V4 (helices E23-n). Finally, an example of a model for an animal mitochondrial SSU rRNA is shown in Fig. 8.

\section{COMPLETENESS, ACCURACY, AND AVAILABILITY OF THE DATA}

SSU rRNA sequences deposited in the GenBank and EMBL nucleotide sequence libraries are obtained weekly from the EMBL file server by electronic mail. By means of an appropriate set of programs, each new sequence is aligned with the most closely related one already present in the alignment, the secondary structure pattern is transposed to the newly aligned sequence, and the complementarity of the postulated secondary structure elements is checked. Manual corrections are made if necessary by means of a specially developed editor. Finally, the newly aligned sequence is automatically compared with the original record in order to eliminate any errors that might have been introduced during editing of the alignment. 
Files containing all the SSU rRNA sequences present in our database are available in the following three formats.

1) The sequences, listed one by one, written continuously without the gaps needed for alignment and without indication of secondary structure elements.

2) The sequences, listed one by one, but with nucleotide symbols interspersed with the gaps necessary for alignment. In this file, each sequence covers 4807 positions, which is the present length of the complete alignment of all eukaryotic, archaeal, bacterial, and organellar sequences.

3) The sequences, listed in the form of an alignment with indication of the secondary elements. The alignment is divided into 49 pages each comprising 100 positions containing a nucleotide or a gap. These positions alternate with an equal number of positions that are either blank or contain a symbol indicating the beginning or end of a secondary structure element. The secondary structure model adopted for each SSU rRNA sequence, is completely defined in this file.

In addition, there are files containing a taxonomic list of species for which the SSU rRNA sequence is known, plus further data as listed in Table 1 of the previous compilation (5) and literature references, and a file containing general documentation on the database.

These files will be made available through 'anonymous ftp' on host uiam3.uia.ac.be (143.169.8.1). The files, as well as later updates, will also be made available to the EMBL nucleotide sequence library at Heidelberg for distribution on their file server and on their CD-ROM disk. Due to the increasing volume of the database, copying it onto diskettes is getting cumbersome. However, researchers who do not have access to the aforementioned distribution channels can address requests for obtaining specific parts of the database on magnetic media to the authors in writing or by sending an electronic mail message to dwachter@reks.uia.ac.be or to rrna@reks.uia.ac.be.

\section{ACKNOWLEDGEMENTS}

Our research is supported by the BRIDGE programme of the Commission of European Communities (contract BIOTCT91-0294), by the Programme on Interuniversity Poles of Attraction of the Office for Science Policy Programming of the Belgian State (contract 23), and by the Fund for Collective Fundamental Research. P.De Rijk is research assistant of the National Fund for Scientific Research.

\section{REFERENCES}

1. Huysmans,E., De Wachter,R. (1986), Nucleic Acids Res. 14, r73-r117.

2. Dams,E., Hendriks,L., Van de Peer,Y., Neefs,J.M., Smits,G., Vandenbempt,I., De Wachter,R. (1988), Nucleic Acids Res. 16, r87-r173.

3. Neefs,J.-M., Van de Peer,Y., Hendriks,L., De Wachter,R. (1990), Nucleic Acids Res. 18, 2237-2317.

4. Neefs,J.-M., Van de Peer,Y., De Rijk,P., Goris,A., De Wachter,R. (1991), Nucleic Acids Res. 19, 1987-2015.

5. De Rijk,P., Neefs,J.-M., Van de Peer,Y., De Wachter,R. (1992), Nucleic Acids Res. 20, 2075-2089.

6. Brusca,R.C., Brusca,G.J. (1990), Invertebrates, Sinauer Associates, Inc., Sunderland.

7. Cronquist,A. (1971), Introductary Botany, Harper \& Row, New York.

8. Ainsworth,G.C., Sparrow,F.K., Sussman,A.S. (1973), The Fungi: an Advanced Treatise, Academic Press, New York, Vol. 4A.

9. Corliss,J.O. (1984), BioSystems 17, 8-126.

10. Woese,C.R. (1987), Microbiol. Rev. 51, 221-271.

11. Woese,C.R. (1991), in Selander, R.K., Clark,A.G., Whittam,T.S. (eds.), Evolution at the Molecular Level. Sinauer Associates, Inc., Sunderland, pp. $1-24$.

12. Olsen,G.J., Woese,C.R. (1993), FASEB. J. 7, 113-123.

13. Holt,J.G. (1984, 1986, 1989) Bergey's Manual of Systematic Bacteriology, Williams \& Wilkins, Baltimore, Vol. 1-4.

14. Van de Peer,Y., Neefs,J.-M., De Wachter,R. (1990), J. Mol. Evol. 30, $463-476$.

15. Jukes,T.H., Cantor,C.R. (1969) in Munro,H.N. (ed.), Mammalian Protein Metabolism. Academic Press, New York, pp. 21-132.

16. Saitou,N., Nei,M. (1987), Mol. Biol. Evol. 4, 406-425.

17. Van de Peer,Y., Neefs,J.-M., De Rijk,P., De Wachter,R. (1993), J. Mol. Evol., in press.

18. Woese,C.R., Gutell,R.R. (1989), Proc. Natl. Acad. Sci. USA 86, 3119-3122.

Table 1. List of species for which the SSU rRNA structure is recorded in the database ${ }^{a}$.

\begin{tabular}{ll}
\hline EUKARYA & Discoglossus pictus \\
ANIMALIA & Eleutherodactylus cuneatus \\
CHORDATA & Gastrophryne carolinensis \\
VERTEBRATA & Grandisonia alternans \\
MAMMALIA & Hyla cinerea \\
Homo sapiens 1 & Ichthyophis bannanicus \\
Homo sapiens 2 & Nesomantis thomasseti \\
Homo sapiens 3 & Plethodon yonhalossee \\
Mus musculus & Scaphiopus holbrooki \\
Oryctolagus cuniculus & Siren intermedia \\
Rattus norvegicus 1 & Typhlonectes natans \\
Rattus norvegicus 2 & Xenopus borealis \\
& Xenopus laevis \\
AVES & \\
Gallus gallus & OSTEICHTYES \\
Turdus migratorius & Fundulus heteroclitus \\
REPTILIA & Latimeria chalumnae \\
Alligator mississippiensis & Sebastolobus altivelis \\
Heterodon platyrhinos & \\
Pseudemys scripta & CHONDRICHTYES \\
Sceloporus undulatus & Echinorhinus cookei \\
AMPHIBIA & Rhinobatos lentiginosus \\
Ambystoma mexicanum & Squalus acanthias \\
Amphiuma tridactylum & AGNATHA \\
Bufo valliceps & Eptatretus stouti \\
& Lampetra aepyptera \\
\end{tabular}


3028 Nucleic Acids Research, 1993, Vol. 21, No. 13

Table 1. continued

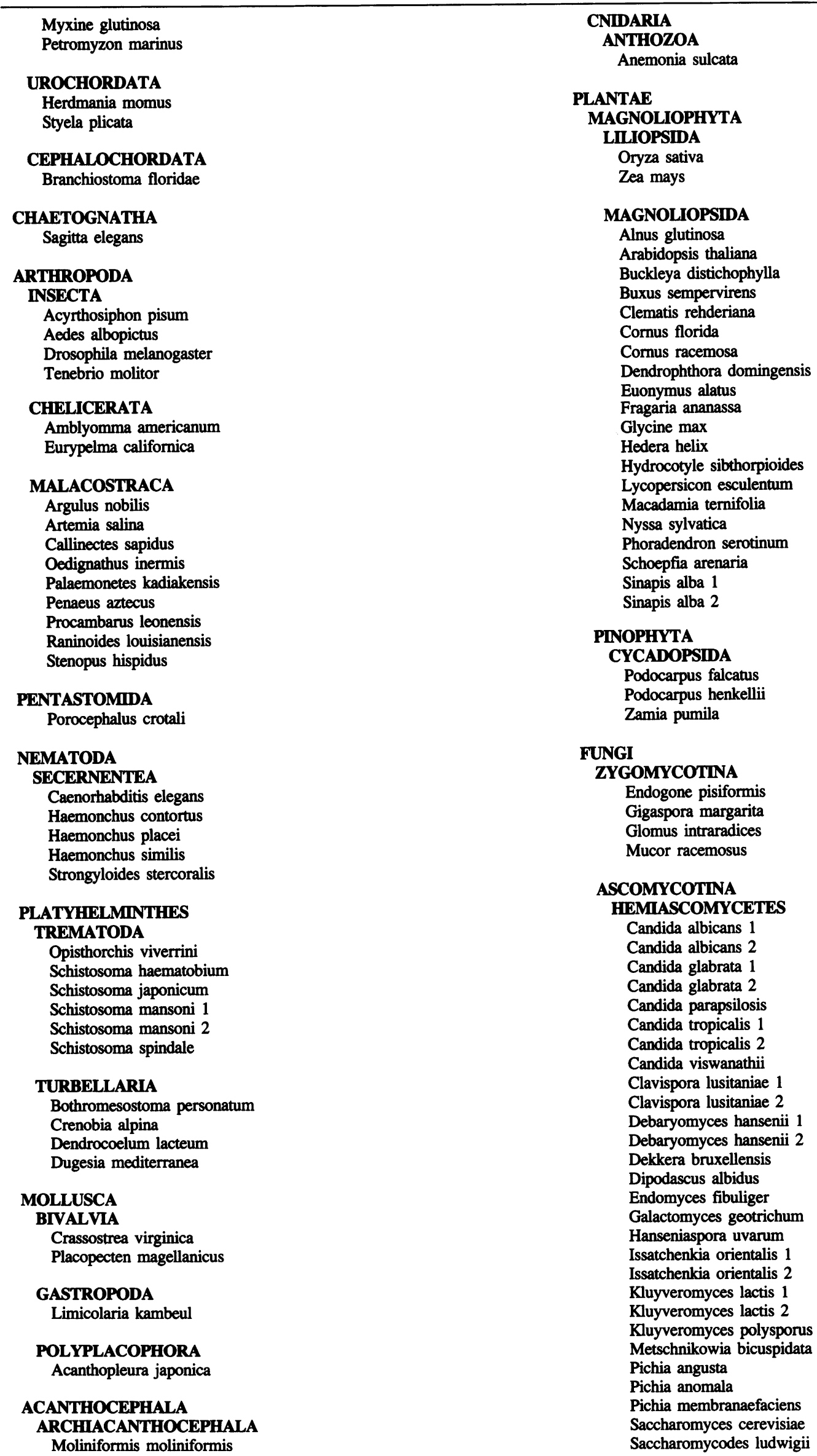


Saccharomycopsis capsularis

Torulaspora delbrueckii

Waltomyces lipofer

Yamadazyma guilliermondii

Yarrowia lipolytica

Zygosaccharomyces rouxii

\section{EUASCOMYCETES}

Ascosphaera apis 1

Ascosphaera apis 2

Aspergillus fumigatus 1

Aspergillus fumigatus 2

Aspergillus fumigatus 3

Aureobasidium pullulans

Byssochlamys nivea

Chaetomium elatum

Coccidioides immitis 1

Coccidioides immitis 2

Emmonsiella capsulata

Eremascus albus

Exophiala dermatidis 1

Exophiala dermatidis 2

Exophiala dermatidis 3

Glomerella cingulata

Hypomyces chrysospermus

Leucostoma persoonii

Monascus purpureus

Neurospora crassa

Ophiostoma schenckii

Ophiostoma stenoceras

Ophiostoma ulmi

Penicilium notatum

Podospora anserina

Pseudallescheria boydii

Sclerotinia sclerotiorum

Sordaria fimicola

Talaromyces flavus

Taphrina deformans

Thermoascus crustaceus

Trichophyton rubrum

UNCERTAIN AFFILIATION

Pneumocystis carinii

Schizosaccharomyces pombe 1

Schizosaccharomyces pombe 2

\section{BASIDIOMYCOTINA \\ USTOMYCETES}

Leucosporidium scottii

Rhodosporidium toruloides 1

Rhodosporidium toruloides 2

Sporobolomyces roseus

Ustilago maydis

TRUE BASIDIOMYCETES

Athelia bombacina

Boletus satanas

Bulleromyces albus

Coprinus cinereus

Cronartium ribicola

Filobasidiella neoformans 1

Filobasidiella neoformans 2

Filobasidiella neoformans 3

Microascus cirrosus

Spongipellis unicolor

Thanatephorus praticola

Trichosporon cutaneum

Xerocomus chrysenteron

\section{PROTISTA}

ASSEMBLAGE CHLOROBIONTS

\section{CHLOROPHYTA}

Ankistrodesmus stipitatus

Asteromonas gracilis

Characium hindaki

Characium perforatum

Characium saccatum
Characium vacuolatum

Chlamydomonas reinhardtii

Chlorella ellipsoidea

Chlorella fusca

Chlorella kessleri

Chlorella lobophora

Chlorella minutissima

Chlorella protothecoides

Chlorella saccharophila

Chlorella sorokiniana

Chlorella vulgaris

Chlorococcopsis minuta

Coleochaete scutata

Coleochaete orbicularis

Dunaliella parva

Dunaliella salina

Friedmannia israeliensis

Hydrodictyon reticulatum

Klebsormidium flaccidum

Nanochlorum eucaryotum

Neochloris aquatica

Neochloris vigenis

Parietochloris pseudoalveolaris

Pediastrum duplex

Prototheca wickerhamii

Prototheca zopfii

Scenedesmus obliquus

Spermatozopsis similis

Tetraselmis striata

Trebouxia impressa

Trebouxia magna

Trebouxia asymmetrica

Volvox carteri

CHAROPHYTA

Chlorokybus atmophyticus

Nitella sp.

\section{ASSEMBLAGE CHROMOBIONTS}

CHRYSOPHYTA

Hibberdia magna

Mallomonas papillosa

Mallomonas striata

Ochromonas danica

Synura spinosa

DINOPHYTA

Alexandrium tamarense

Emiliania huxleyi 1

Emiliania huxleyi 2

Symbiodinium microadriaticum

Symbiodinium pilosum

Symbiodinium sp.

Tribonema aequale

PHAEOPHYTA

Costaria costata

Fucus gardneri

Bacillaria paxillifer

Cylindrotheca closterium

Nannochloropsis salina

Nitzschia apiculata

Rhizosolenia setigera

Skeletonema costatum

Stephanopyxis broschii

\section{ASSEMBLAGE CILIATES}

CILIOPHORA

Blepharisma americanum

Colpidium campylum

Colpoda inflata

Euplotes aediculatus

Glaucoma chattoni

Metopus palaeformis

Onychodromus quadricornutus

Opisthonecta henneguyi

Oxytricha granulifera 
Table 1. continued

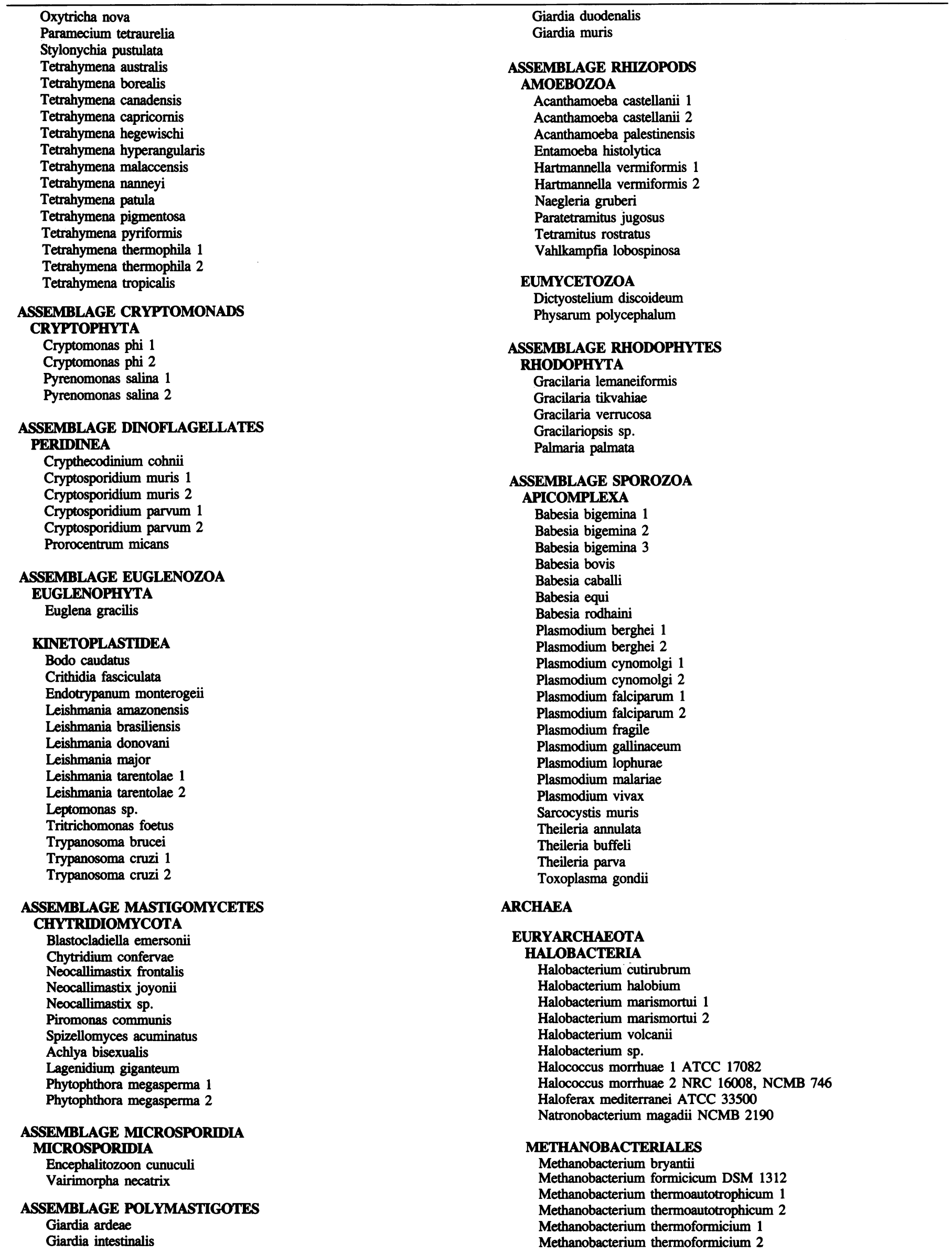


Methanobacterium thermoformicium 3 Methanobacterium thermoformicium 4 Methanobacterium thermoformicium 5 Methanobacterium thermoformicium 6 Methanobacterium thermoformicium 7 Methanobacterium thermoformicium 8 Methanobacterium thermoformicium 9 Methanobrevibacter arboriphilus

Methanosphaera stadtmanii

Methanothermus fervidus 1

Methanothermus fervidus 2

\section{METHANOCOCCALES}

Methanococcus igneus DSM 5666

Methanococcus jannaschii

Methanococcus thermolithotrophicus

Methanococcus voltae

Methanococcus vannielii

\section{METHANOMICROBIUM GROUP}

Methanocorpusculum parvum DSM 3823

Methanogenium cariaci DSM 1497

Methanogenium marisnigri DSM 1498

Methanogenium organophilum DSM 3596

Methanogenium thermophilum DSM 3915

Methanohalophilus mahii DSM 5219

Methanohalophilus zhilinae

Methanohalophilus sp. 1

Methanohalophilus sp. 2 DSM 3243

Methanolobus tindarius DSM 2278

Methanomicrobium mobile DSM 1539

Methanoplanus limicola DSM 2279

Methanosaeta concilii DSM 3013

Methanosaeta thermoacetophila DSM 3870

Methanosarcina acidivorans DSM 2834

Methanosarcina barkeri DSM 1538

Methanosarcina frisia DSM 3318

Methanosarcina frisius

Methanosarcina thermophila DSM 1825

Methanosarcina sp. DSM 4659

Methanospirillum hungatei

Methanothrix soehngenii

Methylcoccoides methylutens DSM 2657

\section{THERMOCOCCALES}

Thermococcus celer DSM 2476

\section{THERMOPLASMA}

Thermoplasma acidophilum

\section{ARCHAEOGLOBALES}

Archaeoglobus fulgidus

\section{METHANOPYRALES}

Methanopyrus kandleri DSM 6324

\section{CRENARCHAEOTA}

Desulfurococcus mobilis

Pyrodictium occultum DSM 2709

Sulfolobus shibatae DSM 5389

Sulfolobus solfataricus

Thermofilum pendens DSM 2475

Thermoproteus tenax

\section{BACTERIA}

\section{PROTEOBACTERIA ALPHA}

Afipia clevelandensis

Afipia felis ATCC 53690

Agrobacterium tumefaciens DSM 30105

Anaplasma marginale

Ancylobacter aquaticus ATCC 25396

Azospirillum lipoferum

Bangasternus orientalis endosymbiont

Bartonella bacilliformis 1 ATCC 35685

Bartonella bacilliformis 2 NCTC 12138

Bartonella bacilliformis 3 ATCC 35685
Beijerinckia indica

Blastobacter denitrificans LMG 8443 (T)

Bradyrhizobium japonicum LMG 6138 (T)

Brucella abortus

Caulobacter bacteroides

Caulobacter crescentus 1

Caulobacter crescentus 2

Caulobacter crescentus 3

Caulobacter subvibrioides

Caulobacter sp. 1

Caulobacter sp. 2

Caulobacter sp. 3

Caulobacter sp. 4

Caulobacter sp. 5

Caulobacter sp. 6

Caulobacter sp. 7

Caulobacter sp. 8

Caulobacter sp. 9

Caulobacter sp. 10

Cowdria ruminantium 1

Cowdria ruminantium 2

Ehrlichia canis 1

Ehrlichia canis 2

Ehrlichia chaftechsis

Ehrlichia equi

Ehrlichia ewingii 1

Ehrlichia ewingii 2

Ehrlichia phagocytophila 1

Ehrlichia phagocytophila 2

Ehrlichia risticii

Ehrlichia sennetsu 1

Ehrlichia sennetsu 2

Erythrobacter longus 1

Erythrobacter longus 2

Erythrobacter longus 3

Erythrobacter sp.

Flavobacterium capsulatum

Flavobacterium devorans

Hirschia baltica

Hyphomicrobium vulgare

Hyphomonas jannaschiana

Hyphomonas sp.

Magnetite-containing magnetic vibrio

Magnetospirillum gryphiswaldense DSM 6361

Magnetospirillum magnetotacticum ATCC 31632

Methylobacterium extorquens 1

Methylobacterium extorquens 2

Methylobacterium organophilum

Methylobacterium sp. 1

Methylobacterium sp. 2

Methylobacterium sp. 3

Methylocystis parvus

Methylosinus sporium

Methylosinus trichosporium

Methylosinus sp. 1

Methylosinus sp. 2

Methylosporovibrio methanicus

Porphyrobacter neustonensis 1

Porphyrobacter neustonensis 2

Pseudomonas diminuta

Rhinocyllus conicus endosymbiont

Rhodobacter capsulatus 1

Rhodobacter capsulatus 2

Rhodobacter sphaeroides 1

Rhodobacter sphaeroides 2

Rhodobacter sphaeroides 3

Rhodomicrobium vannielii

Rhodopseudomonas acidophila

Rhodopseudomonas globiformis

Rhodopseudomonas marina

Rhodopseudomonas palustris

Rhodospirillum centenum

Rhodospirillum fulvum

Rhodospirillum molischianum

Rhodospirillum rubrum

Rhodospirillum salexigenes

Rhodospirillum salinarum 
Table 1. continued

Rhodospirillum sodomense

Rickettsia prowazekii Brienl

Rickettsia rickettsii

Rickettsia typhi Wilmington

Rochalimaea americana

Rochalimaea quintana 1

Rochalimaea quintana 2

Rochalimaea saintelizabethsina

Rochalimaea vinsonii 1

Rochalimaea vinsonii 2

Roseobacter denitrificans 1

Roseobacter denitrificans 2

Simonsiella muelleri

Sitophilus oryzae endosymbiont

Wolbachia pipientis

Zoogloea ramigera

\section{PROTEOBACTERIA BETA}

Alcaligenes eutrophus

Alcaligenes faecalis ATCC 8750 (T)

Alcaligenes xylosoxidans ATCC 15173 (T)

Bordetella bronchiseptica

Chromobacterium fluviatile ATCC 33051 (T)

Chromobacterium violaceum ATCC 12472 (T)

Eikenella corrodens 1 ATCC 23834 (T)

Eikenella corrodens 2 FDC 373

Eikenella corrodens 3 FDC 558

Eikenella corrodens 4 FDC 1073

Eikenella sp.

Gallionella ferruginea

Kingella denitrificans 1 ATCC 33394 (T)

Kingella denitrificans 2

Kingella kingae ATCC 23330 (T)

Kingella orale

Leptothrix discophora

Methylobacillus flagellatum

Methylobacillus glycogenes

Methylomonas methanolica

Methylomonas methylovora

Methylophilus methylotrophus

Neisseria animalis

Neisseria canis

Neisseria denitrificans 1 ATCC 14686 (T)

Neisseria denitrificans 2

Neisseria elongata 1

Neisseria elongata 2

Neisseria flavescens

Neisseria gonorrhoeae 1 NCTC 83785

Neisseria gonorrhoeae 2

Neisseria macaca

Neisseria polysaccharea

Nitrosococcus mobilis

Nitrosolobus multiformis 1

Nitrosolobus multiformis 2

Nitrosomonas europae

Nitrosomonas europaea

Nitrosomonas eutropha

Nitrosovibrio tenuis 1

Nitrosovibrio tenuis 2

Nitrosovibrio tenuis 3

Nitrospira briensis

Pseudomonas cepacia ATCC 25416 (T)

Pseudomonas testosteroni ATCC 11996

Rhodocyclus gelatinosus

Rhodocyclus purpureus

Sphaerotilus natans

Spirillum volutans ATCC 19554 (T)

Thiobacillus thioparus ATCC 8158

Vitreoscilla stercoraria 1

Vitreoscilla stercoraria 2

Zoogloea ramigera 1

Zoogloea ramigera 2

Zoogloea ramigera 3
PROTEOBACTERIA GAMMA*

Acinetobacter calcoaceticus

Alteromonas vaga ATCC 27119

Arhodomonas oleiferhydrans

Bacteroides nodosus

Bathymodiolus thermophilus gill symbiont

Calyptogena magnifica symbiont

Cardiobacterium hominis ATCC 16826 (T)

Chromatium tepidum

Chromatium vinosum ATCC 17899

Codakia orbicularis gill symbiont

Coxiella burnetii

Ectothiorhodospira halochloris

Ectothiorhodospira halophila

Ectothiorhodospira shaposhnikovi

Flavobacterium halmophilum

Flavobacterium lutescens

Halomonas elongata ATCC 33173

Kingella indologenes ATCC 25869 (T)

Legionella bozemanii NCTC 11368 (T)

Legionella erythra NCTC 11977 (T)

Legionella hackeliae NCTC 11979 (T)

Legionella longbeachae NCTC 11477 (T)

Legionella maceachernii NCTC 11982

Legionella micdadei NCTC 11371 (T)

Legionella pneumophila 1 NCTC 11192 (T)

Legionella pneumophila 2 NCTC 11286

Legionella pneumophila 3 NCTC 11233 (T)

Legionella pneumophila 4 NCTC 11405

Legionella pneumophila 5

Legionella spiritensis NCTC 11990 (T)

Legionella sp.

Lucinoma aequizonata gill symbiont

Marinobacter hydrocarbonoclasticus

Methylococcus capsulatus

Methylococcus luteus

Methylomonas albus

Methylomonas methanica

Methylomonas rubra

Methylomonas sp.

Nitrosococcus oceanus 1

Nitrosococcus oceanus 2

Oceanospirillum linum ATCC 11336 (T)

Pseudomonas aeruginosa 1 ATCC 11996

Pseudomonas aeruginosa 2 ATCC 25330

Pseudomonas mendocina

Riftia pachyptila trophosome symbiont

Solemya reidi symbiont

Solemya velum symbiont

Thiobacillus hydrothermalis

Xanthomonas maltophilia

Xylella fastidiosa

\section{PROTEOBACTERIA GAMMA}

Actinobacillus actinomycetemcomitans 1 ATCC 29524

Actinobacillus actinomycetemcomitans 2 ATCC 29523

Actinobacillus actinomycetemcomitans 3 ATCC 33384 (T)

Actinobacillus actinomycetemcomitans 4 FDC Y4

Actinobacillus actinomycetemcomitans 5 ATCC 29522

Actinobacillus capsulatus 1 NCTC 11408 (T)

Actinobacillus capsulatus 2 CCUG 19799

Actinobacillus capsulatus 3 CCUG 12396 (T)

Actinobacillus equuli NCTC 8529 (T)

Actinobacillus hominis

Actinobacillus lignieresii 1 ATCC 19393 (T)

Actinobacillus lignieresii 2 NCTC 4189 (T)

Actinobacillus pleuropneumoniae ATCC 27088 (T)

Actinobacillus salpingitidis

Actinobacillus seminis ATCC 15768 (T)

Actinobacillus suis ATCC 15557

Actinobacillus ureae

Acyrthosyphon pisum symbiont $P$

Acyrthosyphon pisum symbiont $S$ 
Aeromonas caviae 1 NCIMB 13016 (T) Aeromonas caviae 2 ATCC 15467 Aeromonas eucrenophila NCIMB 74 (T)

Aeromonas hydrophila 1

Aeromonas hydrophila 2 ATCC 7966 (T)

Aeromonas jandaei ATCC 49568 (T)

Aeromonas media ATCC 33907 (T)

Aeromonas salmonicida 1 NCIMB 1102 (T)

Aeromonas salmonicida 2 NCIMB 1102

Aeromonas salmonicida 3 NCIMB 1110

Aeromonas schubertii DSM 4882 (T)

Aeromonas sobria NCIMB 12065 (T)

Aeromonas trota ATCC 49657 (T)

Aeromonas veronii NCIMB 13015 (T)

Aeromonas sp. ATCC 35941

Alteromonas haloplanktis ATCC 14393

Arsenophonus nasoniae ATCC 49151 (T)

Barophile 1

Barophile 2

Bisgaard Taxon 2

Bisgaard Taxon 3

Bisgaard Taxon 5

Bisgaard Taxon 6

Bisgaard Taxon 7

Bisgaard Taxon 8

Bisgaard Taxon 9

Bisgaard Taxon 13

Bisgaard Taxon 141

Bisgaard Taxon 142

Buchnera aphidicola 1

Buchnera aphidicola 2

Buchnera aphidicola 3

Buchnera aphidicola 4

Buchnera aphidicola 5

Buchnera aphidicola 6

Buchnera aphidicola 7

Buchnera aphidicola 8

Buchnera aphidicola 9

Buchnera aphidicola 10

Citrobacter freundii

Cryptosaras couesi symbiont

Erwinia carotovora

Erwinia herbicola

Escherichia coli

Euscelidius variegatus parasite

Haemophilus aegyptius NCTC 8502 (T)

Haemophilus aphrophilus ATCC 33389 (T)

Haemophilus ducreyi 1 CIP 542 (T)

Haemophilus ducreyi 2 CIP 542 (T)

Haemophilus ducreyi 3

Haemophilus ducreyi 4 ATCC 27722

Haemophilus haemoglobinophilus NCTC 1659 (T)

Haemophilus haemolyticus NCTC 10659 (T)

Haemophilus influenzae ATCC 33391 (T)

Haemophilus paracuniculus ATCC 29986 (T)

Haemophilus paragallinarum NCTC 11296 (T)

Haemophilus parahaemolyticus NCTC 8479 (T)

Haemophilus parainfluenzae 1 ATCC 7901

Haemophilus parainfluenzae 2 ATCC 33392 (T)

Haemophilus paraphrophaemolyticus NCTC 10670 (T)

Haemophilus paraphrophilus 1 ATCC 29241 (T)

Haemophilus paraphrophilus 2 ATCC 29242

Haemophilus parasuis 1 NCTC 4557 (T)

Haemophilus parasuis 2

Haemophilus segnis ATCC 33393 (T)

Haemophilus somnus

Haemophilus sp.

Haemophilus taxon

Hafnia alvei

Melanocetus johnsoni symbiont

Pasteurella aerogenes ATCC 27883 (T)

Pasteurella anatis ATCC 43329 (T)

Pasteurella avium NCTC 11297 (T)

Pasteurella bett

Pasteurella canis ATCC 43326 (T)
Pasteurella dagmatis ATCC 43325 (T)

Pasteurella gallinarum NCTC 11188 (T)

Pasteurella haemolytica 1 NCTC 10624

Pasteurella haemolytica 2 NCTC 9380 (T)

Pasteurella langaa ATCC 43328 (T)

Pasteurella multocida NCTC 10322 (T)

Pasteurella pneumotropica NCTC 8141 (T)

Pasteurella stomatis ATCC 43327 (T)

Pasteurella testudinis

Pasteurella volantium 1 NCTC 4101

Pasteurella volantium 2 NCTC 3438 (T)

Pasteurella sp. 1 CCUG 19794

Pasteurella sp. 2 CCUG 18782

Photobacterium phosphoreum

Piscirickettsia salmonis

Plesiomonas shigelloides 1

Plesiomonas shigelloides 2 NCIMB 9242 (T)

Proteus vulgaris 1

Proteus vulgaris 2

Ruminobacter amylophilus DSM 1361 (T)

Serratia entomophila ATCC 43705

Serratia ficaria ATCC 33105

Serratia fonticola ATCC 29844

Serratia grimesii ATCC 14460

Serratia liquefaciens ATCC 27592

Serratia marcescens 1 ATCC 13880

Serratia marcescens 2

Serratia odorifera ATCC 33077

Serratia plymuthica ATCC 183

Serratia proteamaculans ATCC 19323

Serratia rubidaea ATCC 27593

Sitophilus zeamais endosymbiont 1

Sitophilus zeamais endosymbiont 2

Thiomicrospira sp.

Vibrio alginolyticus ATCC 17749 (T)

Vibrio anguillarum NCMB 6, ATCC 12964 (T)

Vibrio campbellii ATCC 25920 (T)

Vibrio diazotrophicus ATCC 33466 (T)

Vibrio harveyi 1

Vibrio harveyi 2 ATCC 14126 (T)

Vibrio hollisae ATCC 33564 (T)

Vibrio natriegens ATCC 14048 (T)

Vibrio parahaemolyticus 1

Vibrio parahaemolyticus 2 ATCC 17802 (T)

Vibrio proteolyticus ATCC 15338 (T)

Vibrio vulnificus ATCC 27562 (T)

Wolbachia persica ATCC VR 331

Xenorhabdus nematophilus

Yersinia enterocolitica

\section{PROTEOBACTERIA DELTA}

Angiococcus disciformis

Archangium gephyra

Bdellovibrio bacteriovorus

Bdellovibrio stolpii

Chondromyces apiculatus

Chondromyces crocatus

Cystobacter fuscus

Desulfobacter curvatus DSM 3379

Desulfobacter hydrogenophilus DSM 3380

Desulfobacter lactus DSM 3381

Desulfobacter postgatei DSM 2034

Desulfobacter sp. 1 DSM 2035

Desulfobacter sp. 2 DSM 2057

Desulfobacterium autotrophicum DSM 3382

Desulfobacterium niacini DSM 2650

Desulfobacterium vacuolatum DSM 3385

Desulfobulbus propionicus ATCC 33891

Desulfobulbus sp. DSM 2058

Desulfococcus multivorans ATCC 33890

Desulfomonas pigra ATCC 29098

Desulfomonile tiedjei ATCC 49306

Desulfosarcina variabilis 1

Desulfosarcina variabilis 2 DSM 2060

Desulfovibrio africanus 
Table 1. continued

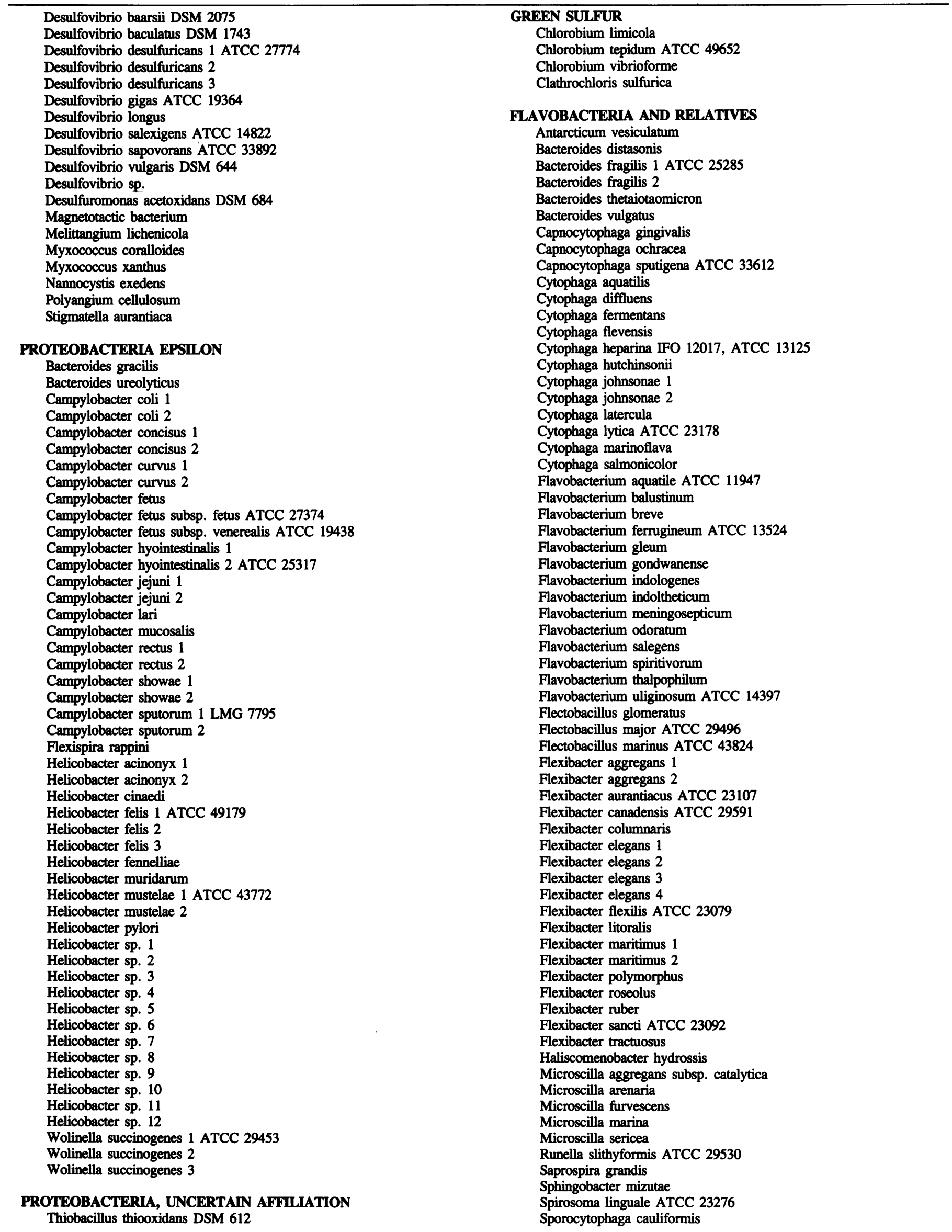


Weeksella virosa

Weeksella zoohelcum

CHLAMYDIAE

Chlamydia pneumoniae

Chlamydia psittaci

Chlamydia trachomatis

\section{FIBROBACTER}

Fibrobacter intestinales 1

Fibrobacter intestinales 2

Fibrobacter intestinales 3

Fibrobacter intestinales 4

Fibrobacter intestinales 5

Fibrobacter succinogenes 1

Fibrobacter succinogenes 2

Fibrobacter succinogenes 3

Fibrobacter succinogenes 4

Fibrobacter succinogenes 5

Fibrobacter succinogenes 6

Fibrobacter succinogenes 7

Fibrobacter succinogenes 8

Fibrobacter succinogenes 9

Fibrobacter succinogenes 10

\section{SPIROCHETES}

Borrelia anserina 1

Borrelia anserina 2

Borrelia burgdorferi

Borrelia burgdorferi 1

Borrelia burgdorferi 2

Borrelia burgdorferi 3

Borrelia burgdorferi 4

Borrelia burgdorferi 5

Borrelia burgdorferi 6

Borrelia burgdorferi 7 ATCC 35210 (T)

Borrelia burgdorferi 8

Borrelia burgdorferi 9

Borrelia burgdorferi 10

Borrelia burgdorferi 11

Borrelia burgdorferi 12

Borrelia coriaceae ATCC 43381

Borrelia hermsii 1 ATCC 35209

Borrelia hermsii 2

Borrelia hermsii 3

Borrelia hermsii 4

Borrelia hermsii 5

Borrelia sp.

Leptonema illini

Leptospira illini

Leptospira interrogans 1 ATCC 23606

Leptospira interrogans 2

Leptospira sp.

Serpula hyodysenteriae 1 ATCC 27164 (T)

Serpula hyodysenteriae 2

Serpula hyodysenteriae 3

Serpula innocens 1 ATCC 29796 (T)

Serpula innocens 2

Spirochaeta aurantia ATCC 25082 (T)

Spirochaeta bajacaliforniensis ATCC 35968 (T)

Spirochaeta halophila ATCC 29478 (T)

Spirochaeta isovalerica

Spirochaeta litoralis ATCC 27000 (T)

Spirochaeta stenostrepta ATCC 25083 (T)

Spirochaeta thermophila DSM 6192

Spirochaeta zuelzeri ATCC 19044 (T)

Spirochaeta sp. 1

Spirochaeta sp. 2

Treponema bryantii ATCC 33254 (T)

Treponema denticola ATCC 33520

Treponema pallidum 1

Treponema pallidum 2

Treponema pectinovorum ATCC 33768 (T)

Treponema phagedenis

Treponema saccharophilum ATCC 43261 (T)

Treponema succinifaciens ATCC 33096 (T)
Treponema sp. 1 ATCC 43811

Treponema sp. 2

Treponema sp. 3

Treponema sp. 4

\section{FUSOBACTERIUM AND RELATIVES}

Bacteroides termitidis

Fusobacterium alocis ATCC 35896 (T)

Fusobacterium gonidiaformans ATCC 25563 (T)

Fusobacterium gonidoformans

Fusobacterium mortiferum 1

Fusobacterium mortiferum 2 ATCC 25557 (T)

Fusobacterium necrogenes ATCC 25556 (T)

Fusobacterium necrophorum ATCC 25286 (T)

Fusobacterium nucleatum

Fusobacterium nucleatum ssp. animalis NCTC 12276 (T)

Fusobacterium nucleatum ssp. fusiformis NCTC 11326 (T)

Fusobacterium nucleatum ssp. nucleatum ATCC 25586 (T)

Fusobacterium nucleatum ssp. polymorphum ATCC 10953

Fusobacterium perfoetens

Fusobacterium periodonticum ATCC 33693 (T)

Fusobacterium russii 1

Fusobacterium russii 2 ATCC 25533 (T)

Fusobacterium simiae 1

Fusobacterium simiae 2 ATCC 33568 (T)

Fusobacterium ulcerans NCTC 12111 (T)

Fusobacterium varium 1

Fusobacterium varium 2 NCTC $10560(T)$

Leptotrichia buccalis

Propionigenium modestum

\section{CYANOBACTERIA}

Anabaena sp.

Anacystis nidulans

Cyanobacterium sp.

Cyanophora paradoxa cyanelle

Mastigocladus laminosus

Prochlorococcus marinus

Prochloron sp.

Prochlorothrix hollandica

GRAM POSITIVES AND RELATIVES, LOW G+C

Acetomaculum ruminis

Acetonema longum DSM 6540 (T)

Acholeplasma entomophilum ATCC 43706

Acholeplasma laidlawii

Acholeplasma modicum ATCC 29102

Acidaminococcus fermentans ATCC 25085

Aerococcus urinae NCTC 12142 (T)

Aerococcus viridans

Alloiococcus otitis

Anaeroplasma abactoclasticum ATCC 27879

Anaeroplasma bactoclasticum ATCC 27112

Anaeroplasma varium ATCC 43167

Asteroleplasma anaerobium ATCC 27880

Bacillus acidocaldarius 1 ATCC 43034

Bacillus acidocaldarius 2 DSM 446 (T)

Bacillus acidoterrestris 1 DSM 3922

Bacillus acidoterrestris 2 DSM 3923

Bacillus alcalophilus DSM 485

Bacillus alvei 1 ATCC 6344

Bacillus alvei 2 NCDO 1153

Bacillus amyloliquefaciens ATCC 23350

Bacillus amylolyticus NCIMB 8144

Bacillus aneurinolyticus NCIMB 10056

Bacillus anthracis

Bacillus atrophaeus NCIMB 12899

Bacillus azotofixans ATCC 35681

Bacillus azotoformans ATCC 29788

Bacillus badius NCDO 1760

Bacillus benzeovorans NCIMB 12555

Bacillus brevis 1 NCIMB 9372

Bacillus brevis 2

Bacillus cereus 1 NCTC 11143

Bacillus cereus 2 NCDO 1771 (T)

Bacillus circulans NCDO 1775 
Table 1. continued

Bacillus coagulans NCDO 1761

Bacillus cycloheptanicus DSM 4006 (T)

Bacillus fastidiosus DSM 91

Bacillus firmus NCIMB 9366

Bacillus globisporus 1 NCIMB 11434

Bacillus globisporus 2 ATCC 23301, DSM 4 (T)

Bacillus gordonae ATCC 29948

Bacillus insolitus DSM 5

Bacillus kaustophilus NCIMB 8547

Bacillus larvae ATCC 9545

Bacillus laterosporus 1 ATCC 6344, DSM 25

Bacillus laterosporus 2 NCDO 1763

Bacillus lautus NCIMB 12780

Bacillus lentimorbus ATCC 14707

Bacillus lentus NCDO 1127

Bacillus licheniformis NCDO 1772

Bacillus macerans 1 ATCC 8244, DSM 24

Bacillus macerans 2 NCDO 1764

Bacillus macquariensis 1 DSM/ATCC 23464 (T)

Bacillus macquariensis 2 NCTC 10419

Bacillus maroccanus NCIMB 10500

Bacillus medusa NCIMB 10437

Bacillus megaterium DSM 32

Bacillus mycoides DSM 2048 (T)

Bacillus pabuli NCIMB 12781

Bacillus pantothenticus NCDO 1765

Bacillus pasteurii NCIMB 8841

Bacillus polymyxa 1 DSM 36

Bacillus polymyxa 2 NCDO 1774

Bacillus popilliae ATCC 14706

Bacillus psychrophilus 1 ATCC 23304

Bacillus psychrophilus 2 ATCC 23304, DSM 3 (T)

Bacillus psychrophilus 3 ATCC 23306, DSM 2273

Bacillus psychrosaccharolyticus ATCC 23296

Bacillus pulvifaciens NCDO 1141

Bacillus pumilus NCDO 1766

Bacillus simplex DSM 1321

Bacillus smithii DSM 4216

Bacillus sphaericus NCDO 1767

Bacillus stearothermophilus 1

Bacillus stearothermophilus 2 NCDO 1768

Bacillus subtilis 1

Bacillus subtilis 2 NCDO 1769

Bacillus thermoglucosadicus ATCC 43742

Bacillus thuringiensis NCIMB 9134 (T)

Brochothrix campestris ATCC 43754 (T)

Brochothrix thermosphacta 1 NCDO 1676 (T)

Brochothrix thermosphacta 2

Carnobacterium divergens NCDO 2763 (T)

Carnobacterium gallinarum

Carnobacterium mobile

Carnobacterium piscicola NCDO 2762 (T)

Clostridium acetobutylicum NCIMB 8052

Clostridium acidiurici

Clostridium aminophilum

Clostridium aminovalericum

Clostridium aurantibutyricum NCIMB 10659

Clostridium barati 1 ATCC 27638

Clostridium barati 2 ATCC 43756

Clostridium barkeri

Clostridium beijerinckii 1 DSM 791

Clostridium beijerinckii 2 NCIMB 9362

Clostridium botulinum 1

Clostridium botulinum 2

Clostridium botulinum 3

Clostridium botulinum 4 NCTC 10281

Clostridium botulinum 5 ATCC 25765

Clostridium butyricum 1 ATCC 43755

Clostridium butyricum 2 DSM 2478

Clostridium butyricum 3 NCIMB 8082

Clostridium cadaveris

Clostridium carnis

Clostridium clostridiiformes

Clostridium coccoides

Clostridium cochlearium
Clostridium estertheticum NCIMB 12511

Clostridium fallax

Clostridium histolyticum

Clostridium innocuum ATCC 14501

Clostridium kluyveri

Clostridium leptum

Clostridium limosum

Clostridium lituseburense

Clostridium ljungdahlii

Clostridium malenominatum

Clostridium mangenotii

Clostridium mayombeii DSM 6539 (T)

Clostridium novyi

Clostridium oceanicum

Clostridium oroticum

Clostridium paraperfringens

Clostridium pasteurianum

Clostridium perfringens 1

Clostridium perfringens 2

Clostridium piliforme

Clostridium purinolyticum

Clostridium quercicolum

Clostridium ramosum ATCC 25582

Clostridium scatologenes

Clostridium sordellii

Clostridium sporogenes

Clostridium sporosphaeroides

Clostridium sticklandii 1

Clostridium sticklandii 2

Clostridium subterminale

Clostridium symbiosum

Clostridium tetanomorphum NCIMB 11547

Clostridium thermaceticum

Clostridium thermoaceticum

Clostridium thermoautotrophicum 1

Clostridium thermoautotrophicum 2

Clostridium thermohydrosulfuricum

Clostridium thermosaccharolyticum

Clostridium thermosulfurogenes

Clostridium tyrobutyricum 1

Clostridium tyrobutyricum 2

Desulfotomaculum nigrificans NCIMB 8395 (T)

Desulfotomaculum orientis NCIMB 8382

Desulfotomaculum ruminis NCIMB 8452

Enterococcus avium NCDO 2369 (T)

Enterococcus casseliflavus NCDO 2376

Enterococcus columbae NCIMB 13013

Enterococcus dispar

Enterococcus durans NCDO 596 (T)

Enterococcus faecalis

Enterococcus faecium NCDO 942 (T)

Enterococcus gallinarum NCDO 2313 (T)

Enterococcus hirea NCDO 1258 (T)

Enterococcus malodoratus NCDO 846 (T)

Enterococcus mundtii NCDO 2375 (T)

Enterococcus pseudoavium NCDO 2138 (T)

Enterococcus raffinosus NCTC 12192 (T)

Enterococcus sulfureus NCDO 2379

Epulopiscium sp. 1

Epulopiscium sp. 2

Erysipelothrix rhusiopathiae ATCC 19414

Eubacterium alactolyticum DSM 3980

Eubacterium biforme

Eubacterium limosum

Eubacterium tenue

Gemella haemolysans

Heliobacterium chlorum

Kurthia zopfii

Lactobacillus acetolerans

Lactobacillus acidophilus 1 NCDO 1748 (T)

Lactobacillus acidophilus 2

Lactobacillus agilis

Lactobacillus alimentarius

Lactobacillus amylophilus

Lactobacillus amylovorus 
Nucleic Acids Research, 1993, Vol. 21, No. 133037

Lactobacillus animalis 1 NCDO 2425 (T)

Lactobacillus animalis 2

Lactobacillus aviarius

Lactobacillus bifermentans

Lactobacillus brevis 1 NCDO 1749 (T)

Lactobacillus brevis 2

Lactobacillus buchneri 1 NCDO 110 (T)

Lactobacillus buchneri 2

Lactobacillus carnis

Lactobacillus casei 1

Lactobacillus casei 2 NCDO 161 (T)

Lactobacillus casei 3

Lactobacillus catenaforme ATCC 25536 (T)

Lactobacillus confusus 1 NCDO 5186

Lactobacillus confusus 2 DSM 20196

Lactobacillus coryniformis

Lactobacillus delbrueckii 1

Lactobacillus delbrueckii 2 NCDO 213

Lactobacillus divergens

Lactobacillus farciminis

Lactobacillus fermentum 1 NCDO 1750 (T)

Lactobacillus fermentum 2

Lactobacillus fructivorans

Lactobacillus fructosus NCDO 2345 (T)

Lactobacillus gasseri 1 NCDO 2233 (T)

Lactobacillus gasseri 2

Lactobacillus halotolerans DSM 20190

Lactobacillus helveticus NCDO 2712 (T)

Lactobacillus hilgardii

Lactobacillus kandleri 1 NCDO 2753

Lactobacillus kandleri 2 DSM 20593

Lactobacillus lactis

Lactobacillus mali

Lactobacillus maltaromicus 1

Lactobacillus maltaromicus 2 JCM 1154

Lactobacillus minor 1 NCDO 1973

Lactobacillus minor 2 DSM 20014

Lactobacillus murinus

Lactobacillus oris NCDO 2160 (T)

Lactobacillus plantarum 1

Lactobacillus plantarum 2 NCDO 1752

Lactobacillus ruminis

Lactobacillus sake

Lactobacillus salivarius

Lactobacillus sanfrancisco $1 \mathrm{JCM} 5668$

Lactobacillus sanfrancisco 2

Lactobacillus sharpeae

Lactobacillus thermophilus

Lactobacillus vaginalis NCTC 12197

Lactobacillus vermiforme

Lactobacillus viridescens 1 NCDO 1655

Lactobacillus viridescens 2 ATCC 12706

Lactobacillus vitulinus ATCC 27783

Lactobacillus sp.

Lactococcus garviae NCDO 2156

Lactococcus lactis $1 \mathrm{ssp}$. lactis NCDO 2118

Lactococcus lactis 2

Lactococcus piscium

Lactococcus plantarum NCDO 1871

Lactococcus raffinolactis NCDO 2112

Leuconostoc amelobiosum

Leuconostoc carnosum

Leuconostoc citreum NCDO 1837

Leuconostoc cremoris DSM 20346

Leuconostoc fallax DSM 20189

Leuconostoc gelidum

Leuconostoc lactis 1 DSM 20202

Leuconostoc lactis 2 NCDO 533

Leuconostoc mesenteroides 1 DSM 20343

Leuconostoc mesenteroides 2 NCDO 523

Leuconostoc oenos 1 DSM 20252

Leuconostoc oenos 2 NCDO 1674

Leuconostoc paramesenteroides 1 DSM 20288

Leuconostoc paramesenteroides 2 NCDO 803

Leuconostoc pseudomesenteroides NCDO 768

Listeria grayi CIP 6818 (T)

Listeria innocua NCTC $11288\left(T^{\circ}\right.$
Listeria ivanovii NCTC 11846 (T)

Listeria monocytogenes 1

Listeria monocytogenes 2 NCTC 10357 (T)

Listeria murrayi NCTC 10812 (T)

Listeria seeligeri NCTC 11856 (T)

Listeria welshimeri NCTC 11857 (T)

Marinococcus halophilus NCIMB 2178 (T)

Megasphaera elsdenii

Mycoplasma agalactiae NCTC 10123

Mycoplasma arginini ATCC 23838

Mycoplasma arthritidis ATCC 19611

Mycoplasma bovigenitalium ATCC 19852

Mycoplasma californicum ATCC 33461

Mycoplasma capricolum

Mycoplasma coragypsum

Mycoplasma ellychniae ATCC 43707

Mycoplasma fermentans ATCC 19989

Mycoplasma flocculare ATCC 27716

Mycoplasma gallisepticum 1

Mycoplasma gallisepticum 2

Mycoplasma gallisepticum 3

Mycoplasma hominis 1 ATCC 23114

Mycoplasma hominis 2

Mycoplasma hyopneumoniae ATCC 27719

Mycoplasma hyorhinis ATCC 17981

Mycoplasma iowae 1 ATCC 33552

Mycoplasma iowae 2

Mycoplasma-like organism 1

Mycoplasma-like organism 2

Mycoplasma-like organism 3

Mycoplasma lipophilum ATCC 27104

Mycoplasma mobile ATCC 43663

Mycoplasma muris ATCC 33757

Mycoplasma mycoides

Mycoplasma neurolyticum ATCC 19988

Mycoplasma orale ATCC 23714

Mycoplasma pirum ATCC 25960

Mycoplasma pneumoniae ATCC 15531

Mycoplasma pulmonis ATCC 19612

Mycoplasma putrefaciens ATCC 15718

Mycoplasma salivarium ATCC 23064

Mycoplasma sualvi ATCC 33004

Mycoplasma sp. 1

Mycoplasma sp. 2

Mycoplasma sp. 3 ATCC 49193

Mycoplasma sp. 4 ATCC 49191

Oenothera hookeri MLO

Pectinatus cerevisiiphilus ATCC 29359

Pectinatus frisigensis ATCC 33332

Pediococcus acidilactici

Pediococcus pentosaceus

Peptococcus niger DSM 20475 (T)

Peptostreptococcus anaerobius

Planococcus citreus NCIMB 1493 (T)

Planococcus kocurii NCIMB 629

Quinella ovalis

Selenomonas lacticifex DSM 20757

Selenomonas ruminantium 1 subsp. lactilytica DSM 2872

Selenomonas ruminantium 2

Selenomonas ruminantium 3

Selenomonas sputigena ATCC 35185

Spiroplasma ATCC 43302

Spiroplasma apis ATCC 33834

Spiroplasma citri 1 ATCC 27556

Spiroplasma citri 2

Spiroplasma mirum ATCC 29335

Spiroplasma sp. 1 ATCC 43153

Spiroplasma sp. 2 ATCC 33827

Spiroplasma sp. 3 ATCC 33835

Spiroplasma sp. 4 ATCC 33825

Spiroplasma sp. 5 ATCC 43210

Spiroplasma sp. 6 ATCC 43525

Sporolactobacillus inulinus

Sporomusa paucivorans

Sporomusa termitida

Sporosarcina halophila NCIMB 2269

Sporosarcina ureae NCIB 9251 
Table 1. continued

Staphylococcus aureus NCDO 949

Staphylococcus muscae CCM 4175 (T)

Staphylococcus schleiferi DSM 4807 (T)

Staphylococcus sciuri

Streptococcus acidominimus NCD̄O 2025 (T)

Streptococcus agalactiae NCDO 1348 (T)

Streptococcus alactolytiens NCDO 1091 (T)

Streptococcus anginosus NCTC 10713 (T)

Streptococcus bovis 1 NCDO 597 (T)

Streptococcus bovis 2

Streptococcus canis DSM 20715 (T)

Streptococcus cecorum NCDO 2674 (T)

Streptococcus constellatus NCTC 11325 (T)

Streptococcus cremoris

Streptococcus criae NCDO 2772 (T)

Streptococcus cricetus NCDO 2720 (T)

Streptococcus downei NCTC 11391 (T)

Streptococcus dysgalactiae NCDO 2023 (T)

Streptococcus equi NCDO 2493 (T)

Streptococcus equinus NCDO 1037 (T)

Streptococcus hansenii

Streptococcus hyointestinalis DSM 20770 (T)

Streptococcus intermedius NCTC 11324 (T)

Streptococcus lactis

Streptococcus macacae NCTC 11558 (T)

Streptococcus mutans NCTC 10449 (T)

Streptococcus oralis NCTC 11427 (T)

Streptococcus parasanguis NCTC 7863 (T)

Streptococcus parauberis NCDO 651

Streptococcus pleomorphus ATCC 29734

Streptococcus pneumoniae NCTC 7465 (T)

Streptococcus porcinus NCDO 600 (T)

Streptococcus pyogenes NCDO 2381 (T)

Streptococcus rattus NCDO 2723 (T)

Streptococcus saccharolyticus NCDO 2594

Streptococcus salivarius 1 NCDO 1779 (T)

Streptococcus salivarius 2

Streptococcus sanguis NCTC 7863

Streptococcus sobrinus DSM 20742 (T)

Streptococcus suis NCTC 10237 (T)

Streptococcus thermophilus NCDO 573 (T)

Streptococcus uberis NCDO 643

Streptococcus vestibularis NCTC 12166 (T)

Syntrophomonas wolfei

Syntrophospora bryantii

Ureaplasma urealyticum NCTC 10177

Vagococcus fluvialis NCDO 2497

Vagococcus salmoninarum

Western aster yellow MLO isolated from celery

Zymophilus paucivorans DSM 20756

GRAM POSITIVES AND RELATIVES, HIGH G+C

Actinomyces bovis 1 DSM 43014

Actinomyces bovis 2 DSM 43014

Actinomyces israelii 1 DSM 43020

Actinomyces israelii 2 DSM 43020

Actinomyces naeslundii 1 DSM 43013

Actinomyces naeslundii 2 DSM 43013

Actinomyces odontolyticus 1 DSM 43331

Actinomyces odontolyticus 2 DSM 43331

Actinomyces pyogenes ATCC 19411

Actinomyces viscosus 1 DSM 43027

Actinomyces viscosus 2 DSM 43027

Actinopolyspora halophila ATCC 279

Aeromicrobium erythreum NRRL B-3381 (T)

Amycolata autotrophica DSM 43210

Amycolata nitrificans DSM new

Amycolata petroleophila DSM new

Amycolatopsis azurae NRRL 11412

Amycolatopsis fastidiosa ATCC 31181

Amycolatopsis methanolica NCIB 11946 (T)

Arthrobacter globiformis

Arthrobacter simplex ATCC 6946

Arthrobacter sp.

Atopobium parvulum
Atopobium rimae

Bifidobacterium adolescentis

Bifidobacterium asteroides

Bifidobacterium bifidum

Bifidobacterium breve

Bifidobacterium catenulatum

Bifidobacterium coryneforme

Bifidobacterium cunniculi

Bifidobacterium dentium

Bifidobacterium globosum

Bifidobacterium indicum

Bifidobacterium infantis

Bifidobacterium longum

Bifidobacterium magnum

Bifidobacterium minimum

Bifidobacterium pseudolongum

Bifidobacterium suis

Clavibacter xyli

Corynebacterium renale ATCC 19412

Corynebacterium variabilis NCIB 9455, NCDO 2097 (T)

Corynebacterium xerosis

Dermatophilus congolensis

Eubacterium suis DSM 20639, ATCC 33144 (T)

Faenia rectivirgula ATCC 33515 (T)

Frankia sp. 1

Frankia sp. 2

Frankia sp. 3

Frankia sp. 4

Frankia sp. 5

Fusobacterium prausnitzii

Gardnerella vaginalis

Gordona terrae DSM 43249

Kibdelosporangium aridum ATCC 39323

Kitasatosporia griseola NRRL B-16229

Kitasatosporia phosalacinea NRRL B-16230

Kitasatosporia setae NRRL B-16185

Lactobacillus minutus

Micrococcus luteus

Mobiluncus curtisii ATCC 35421

Mobiluncus mulieris ATCC 35423

Mycobacterium aichiense ATCC 27280

Mycobacterium asiaticum 1 ATCC 25276

Mycobacterium asiaticum 2 ATCC 25276

Mycobacterium aurum 1 ATCC 23366

Mycobacterium aurum 2 ATCC 23366

Mycobacterium avium 1

Mycobacterium avium 2

Mycobacterium avium 3 DSM 43216

Mycobacterium avium 4

Mycobacterium avium 5

Mycobacterium avium 6

Mycobacterium avium 7

Mycobacterium avium 8

Mycobacterium avium 9

Mycobacterium avium 10

Mycobacterium bovis 1

Mycobacterium bovis 2

Mycobacterium chelonae ATCC 14472

Mycobacterium chelonei ATCC 19977

Mycobacterium chitae 1 ATCC 19627

Mycobacterium chitae 2

Mycobacterium chitae 3 ATCC 19627

Mycobacterium chitae 4 ATCC 19627

Mycobacterium chubuense ATCC 27278

Mycobacterium confluentis

Mycobacterium cookii ATCC 49103, DSM 43922 (T)

Mycobacterium diernhoferi ATCC 19340

Mycobacterium fallax ATCC 35219

Mycobacterium farcinogenes DSM 43294

Mycobacterium flavescens 1 ATCC 14474

Mycobacterium flavescens 2 ATCC 14474

Mycobacterium fortuitum ATCC 6841 (T)

Mycobacterium gadium 1

Mycobacterium gadium 2 ATCC 27726

Mycobacterium gastri ATCC 15754 
Mycobacterium gilvum ATCC 43909 Mycobacterium gordonae 1 ATCC 14470 Mycobacterium gordonae 2 ATCC 14470 Mycobacterium intracellulare 1 ATCC 15985 Mycobacterium intracellulare 2

Mycobacterium intracellulare 3 Mycobacterium intracellulare 4 Mycobacterium intracellulare 5 Mycobacterium intracellulare 6 Mycobacterium kansasii 1 DSM 43224 Mycobacterium kansasii 2 ATCC 12478 Mycobacterium komossense ATCC 33013 Mycobacterium leprae 1

Mycobacterium leprae 2

Mycobacterium leprae 3

Mycobacterium madagascariense

Mycobacterium malmoense 1 ATCC 29571

Mycobacterium malmoense 2

Mycobacterium marinum

Mycobacterium neoaurum ATCC 25795

Mycobacterium nonchromogenicum 1 ATCC 19530

Mycobacterium nonchromogenicum 2 ATCC 19530

Mycobacterium obuense ATCC 27023

Mycobacterium paratuberculosis 1

Mycobacterium paratuberculosis 2

Mycobacterium paratuberculosis 3

Mycobacterium paratuberculosis 4

Mycobacterium paratuberculosis 5

Mycobacterium paratuberculosis 6

Mycobacterium paratuberculosis 7

Mycobacterium paratuberculosis 8

Mycobacterium paratuberculosis 9 ATCC 19698

Mycobacterium phlei ATCC 11758

Mycobacterium scrofulaceum ATCC 19981

Mycobacterium senegalense ATCC 35796

Mycobacterium simiae ATCC 25275

Mycobacterium smegmatis ATCC 14468

Mycobacterium sphagni ATCC 33026

Mycobacterium szulgai 1 ATCC 25799

Mycobacterium szulgai 2

Mycobacterium terrae 1 ATCC 15755

Mycobacterium terrae 2 ATCC 15755

Mycobacterium thermoresistible 1 ATCC 19527

Mycobacterium thermoresistible 2 ATCC 19527

Mycobacterium triviale ATCC 23292

Mycobacterium tuberculosis 1

Mycobacterium tuberculosis 2

Mycobacterium tuberculosis 3 NCTC 7416

Mycobacterium ulcerans

Mycobacterium vaccae ATCC 15483

Mycobacterium xenopi 1 ATCC 19250

Mycobacterium xenopi 2 ATCC 19250

Mycobacterium sp. 1

Mycobacterium sp. 2

Mycobacterium sp. 3

Nocardia asteroides 1 DSM 43005

Nocardia asteroides 2 ATCC 3306

Nocardia otitidis-caviarum

Nocardioides albus DSM 43109

Nocardioides fastidiosa NCIB 12713 (T)

Nocardioides jensenii DSM 29641

Nocardioides luteus NCIB 11455

Nocardioides simplex 1 NCIB 8929

Nocardioides simplex 2 NCIB 8929 (T)

Propionibacterium acidi-propionici DSM 20272

Propionibacterium acnes 1 DSM 1897

Propionibacterium acnes 2

Propionibacterium freudenreichii DSM 20271

Propionibacterium jensenii DSM 20535

Propionibacterium propionicus DSM 43307

Propionibacterium thoenii DSM 20276

Pseudonocardia thermophila 1 ATCC 19285 (T)

Pseudonocardia thermophila 2 ATCC 19285 (T)

Renibacterium salmoninarum ATCC 33209 (T)

Rhodococcus equi Equine lung

Rhodococcus erythropolis DSM 43188

Rhodococcus fascians DSM 2013
Rothia dentocariosa

Saccharomonospora viridis ATCC 15386

Saccharopolyspora erythraea NRRL 2338

Saccharopolyspora hirsuta 1 ATCC 27875 (T)

Saccharopolyspora hirsuta 2

Saccharopolyspora hordei

Saccharothrix australiensis ATCC 31947

Streptomyces albus DSM 40313, ISP 5313 (T)

Streptomyces ambofaciens ATCC 23877

Streptomyces brasiliensis DSM 43159 (T)

Streptomyces coelicolor 1

Streptomyces coelicolor 2

Streptomyces diastaticus DSM 40496, ISP 5496 (T)

Streptomyces griseus subsp. griseus ATCC 10137

Streptomyces lavendulae DSM 2014, ISP 5069 (T)

Streptomyces lividans

Streptomyces purpureus DSM 43460 (T)

Streptoverticillium abikoense DSM 40831 (T)

Streptoverticillium baldacii

Streptoverticillium cinnamoneum spp. azacolutum

Streptoverticillium cinnamoneum spp. cinnamoneum

Streptoverticillium ladakanum DSM 40587 (T)

Streptoverticillium luteoreticuli

Streptoverticillium olivoreticuli ssp. cellulophilum

Streptoverticillium salmonis

Terrabacter tumescens NCIB 8914

Tropheryma whippelii

Tsukamurella aurantiacus NCTC 10741

Tsukamurella paurometabolum 1 DSM 20162, NCTC 10741

Tsukamurella paurometabolum 2 NCTC 10741

GREEN NON SULFUR

Chloroflexus aurantiacus

Herpetosiphon aurantiacus ATCC 23779

Sphaerobacter thermophilus DSM 20745 (T)

Thermomicrobium roseum ATCC 27502

\section{RADIORESISTANT MICROCOCCI AND RELATIVES}

Deinococcus radiodurans UWO 298

Thermus aquaticus 1

Thermus aquaticus 2

Thermus aquaticus 3 ATCC 27978

Thermus filiformis 1 ATCC 43280

Thermus filiformis 2

Thermus flavus 1 ATCC 33923

Thermus flavus 2

Thermus ruber 1 ATCC 35948

Thermus ruber 2

Thermus thermophilus 1

Thermus thermophilus 2 ATCC 27634

Thermus thermophilus 3 ATCC 27634

Thermus thermophilus 4

Thermus sp. 1

Thermus sp. 2

Thermus sp. 3

Thermus sp. 4

Thermus sp. 5

Thermus sp. 6

Thermus sp. 7

Thermus sp. 8

Thermus sp. 9

Thermus sp. 10

\section{THERMOTOGALES}

Fervidobacterium nodosum ATCC 35602

Fervidobacterium islandicum DSM 5733 (T)

Thermosipho africanus

Thermotoga maritima DSM 3109

PLANCTOMYCES AND RELATIVES

Gemmata obscuriglobus 1 UQM 2246

Gemmata obscuriglobus 2 UQM 2246

Isosphaera pallida

Pirellula marina DSM 3645

Planctomyces limnophilus ATCC 43296

Planctomyces maris ATCC 29209 (T)

Planctomyces staleyi 
Table 1. continued

\begin{tabular}{|c|c|}
\hline UNCERTAIN AFFUIATION & Antilocapra americana \\
\hline Aquifex pyrophilus & Atelerix albiventris \\
\hline Flexistipes sinusarabici DSM 4947 & Balaenoptera physalus \\
\hline Haloanaerobium praevalens & Bathyergus janetta \\
\hline Leptospirillum sp. DSM 2391 & Bathyergus suillus \\
\hline Sporohalobacter lortetii & Blarina brevicauda \\
\hline Synergistis jonesii & $\begin{array}{l}\text { Bos taurus } \\
\text { Boselaphus tragocamelus }\end{array}$ \\
\hline PLASTIDS & $\begin{array}{l}\text { Capra hircus } \\
\text { Cephalophus maxwelli }\end{array}$ \\
\hline PLANTAE & Cervus unicolor \\
\hline BRYOPHYTA & Cryptomys hottentotus 1 \\
\hline MARCHANTIOPSIDA & Cryptomys hottentotus 2 \\
\hline Marchantia polymorpha & $\begin{array}{l}\text { Cryptomys hottentotus damarensis } \\
\text { Damaliscus dorcas }\end{array}$ \\
\hline MAGNOLIOPHYTA & Gazella thomsoni \\
\hline LILIOPSIDA & Georychus capensis \\
\hline Oryza sativa & Gorilla gorilla \\
\hline Zea mays & Heliophobius argenteocinereus \\
\hline MAGNOLIOPSIDA & Heterocephalus glaber \\
\hline Conopholis americana & Homo sapiens 1 \\
\hline Epifagus virginiana & Homo sapiens 2 \\
\hline Glycine $\max$ & Hydropotes inermis \\
\hline Nicotiana plumbaginifolia & Kobus ellipsiprymnus \\
\hline Nicotiana tabacum 1 & Madoqua kirki \\
\hline Nicotiana tabacum 2 & Muntiacus reevesi \\
\hline Pisum sativum 1 & Mus musculus \\
\hline Pisum sativum 2 & Odocoileus virginianus \\
\hline & Oryx gazella \\
\hline $\begin{array}{l}\text { PROTISTA } \\
\text { ASSEMBLAGE CHLOROBIONTS }\end{array}$ & Pan paniscus \\
\hline ASSEMBLAGE CHLOROBIONTS & $\begin{array}{l}\text { Pan troglodytes } \\
\text { Petromus typicus }\end{array}$ \\
\hline $\begin{array}{l}\text { CHLOROPHYTA } \\
\text { Chlamydomonas eugametos }\end{array}$ & $\begin{array}{l}\text { Petromus typicus } \\
\text { Phoca vitulina }\end{array}$ \\
\hline $\begin{array}{l}\text { Chlamydomonas eugametos } \\
\text { Chlamydomonas moewusii }\end{array}$ & $\begin{array}{l}\text { Phoca vitulina } \\
\text { Pongo pigmaeus }\end{array}$ \\
\hline Chlamydomonas moewusii & $\begin{array}{l}\text { Pongo p1gmaeus } \\
\text { Rattus norvegicus } 1\end{array}$ \\
\hline Chlamydomonas reinhardtii & Rattus norvegicus 2 \\
\hline Chlorella ellipsoidea & Thryonomys swinderianus \\
\hline Chlorella kessleri & Tragelaphus imberbis \\
\hline Chlorella mirabilis & Tragulus napu \\
\hline Chlorella protothecoides & AVES \\
\hline Chlorella sorokiniana & $\begin{array}{l}\text { AVES } \\
\text { Coturnix japonica }\end{array}$ \\
\hline Chlorella vulgaris & $\begin{array}{l}\text { Coturnix japonica } \\
\text { Gallus gallus }\end{array}$ \\
\hline ASSEMBLAGE CHROMOBIONTS & \\
\hline CHRYSOPHYTA & AMPHIBIA \\
\hline Ochromonas danica & Rana catesbeiana \\
\hline Olisthodiscus luteus & Xenopus laevis \\
\hline HAPTOPHYTA & OSTEICHTYES \\
\hline Ochrosphaera spec. & $\begin{array}{l}\text { Crossostoma lacustre } \\
\text { Cyprinus carpio }\end{array}$ \\
\hline PHAEOPHYTA & \\
\hline Pylaiella littoralis 1 & ECHINODERMATA \\
\hline & ECHINOIDEA \\
\hline ASSEMBLAGE CRYPTOMONADS & Paracentrotus lividus \\
\hline CRYPTOPHYTA & Strongylocentrotus purpuratus \\
\hline Cryptomonas phi & \\
\hline Pyrenomonas salina & $\begin{array}{l}\text { ARTHROPODA } \\
\text { INSECTA }\end{array}$ \\
\hline ASSEMBLAGE EUGLENOZOA & Apis mellifera ligustica \\
\hline EUGLENOPHYTA & Drosophila virilis \\
\hline Astasia longa & Drosophila yakuba \\
\hline Euglena gracilis & NEMATODA \\
\hline ASSEMBLAGE RHODOPHYTES & SECERNENTEA \\
\hline RHODOPHYTA & Ascaris suum \\
\hline Antithamnion sp. & Caenorhabditis elegans \\
\hline Cyanidium caldarium & PLANTAE \\
\hline MITOCHONDRIA & $\begin{array}{l}\text { BRYOPHYTA } \\
\text { MARCHANTIOPSIDA }\end{array}$ \\
\hline ANIMALIA & Marchantia polymorpha \\
\hline CHORDATA & \\
\hline VERTEBRATA & MAGNOLIOPHYTA \\
\hline MAMMALIA & LILIOPSIDA \\
\hline Aepyceros melampus & Secale cereale \\
\hline Amblysomus hottentotus & Triticum aestivum \\
\hline
\end{tabular}


Zea diploperennis

Zea mays 1

Zea mays 2

\section{MAGNOLIOPSIDA}

Glycine max

Lupinus luteus

Oenothera sp.

\section{FUNGI}

ASCOMYCOTINA

HEMIASCOMYCETES

Saccharomyces cerevisiae 1

Saccharomyces cerevisiae 2

Saccharomyces cerevisiae 3

Saccharomyces cerevisiae 4

Saccharomyces cerevisiae 5

EUASCOMYCETES

Aspergillus nidulans

Podospora anserina

\section{UNCERTAIN AFFILIATION}

Schizosaccharomyces pombe

\section{PROTISTA}

ASSEMBLAGE CHLOROBIONTS

CHLOROPHYTA

Chlamydomonas reinhardtii

Prototheca wickerhamii

\section{ASSEMBLAGE CILIATES \\ CILIOPHORA}

Paramecium aurelia

Paramecium primaurelia

Paramecium tetraurelia

Tetrahymena pyriformis 1

Tetrahymena pyriformis 2

\author{
ASSEMBLAGE EUGLENOZOA \\ KINETOPLASTIDS \\ Crithidia fasciculata \\ Leishmania tarentolae \\ Leptomonas sp. \\ Trypanosoma brucei \\ ASSEMBLAGE SPOROZOA \\ APICOMPLEXA \\ Plasmodium falciparum 1 \\ Plasmodium falciparum 2
}

(a) Some species names are listed several times followed by a sequential number. This means that the SSU rRNA sequence has been determined several times, usually by different authors. The sequences are not necessarily the same since they may have been determined for different varieties or strains of a species, or for different genes of the same organism. The systematics followed for the three domains are mentioned in the text. Plastidial and mitochondrial structures are listed according to the systematics followed for the host organism. In the case of Archaea and Bacteria, the species name is followed by the culture collection name and number if specified by the author. This number is followed by (T) if it is a type species. Abbreviations of culture collection names can be found in the catalogs of the American Type Culture Collection (ATCC), Deutsche Sammlung von Mikroorganismen (DSM), and Laboratorium voor Microbiologie Universiteit Gent (LMG). The assignment of the archaeal and bacterial species to taxa is based on the tree shown in outline in Fig. 1. These taxa correspond to the divisions and subdivisions distinguished by Woese and coworkers $(10-12)$, except for the bacterial genera Fibrobacter and Fusobacterium, which form separate clusters and therefore are listed as separate taxa. The taxon of Gram positive bacteria of low GC contents and relatives comprises a number of genera with gram negative cell walls: Megasphaera, Pectinatus, Selenomonas, Sporomusa, and Zymophilus. For the Archaea, the classification is slightly different from that followed by Olsen and Woese (12), as explained in the text.

(b) Most of the species listed under the heading 'Proteobacteria gamma*' are attributed to the Proteobacteria $\gamma$ group by Woese (11), although they cluster with the Proteobacteria $\beta$ in the tree of Fig. 1. Exceptions are Xanthomonas maltophilia and Xylella fastidiosa which belong to the Proteobacteria $\beta$ group according to the same author (11).

Table 2. Helix occupancy in eukaryotic SSU rRNAs.

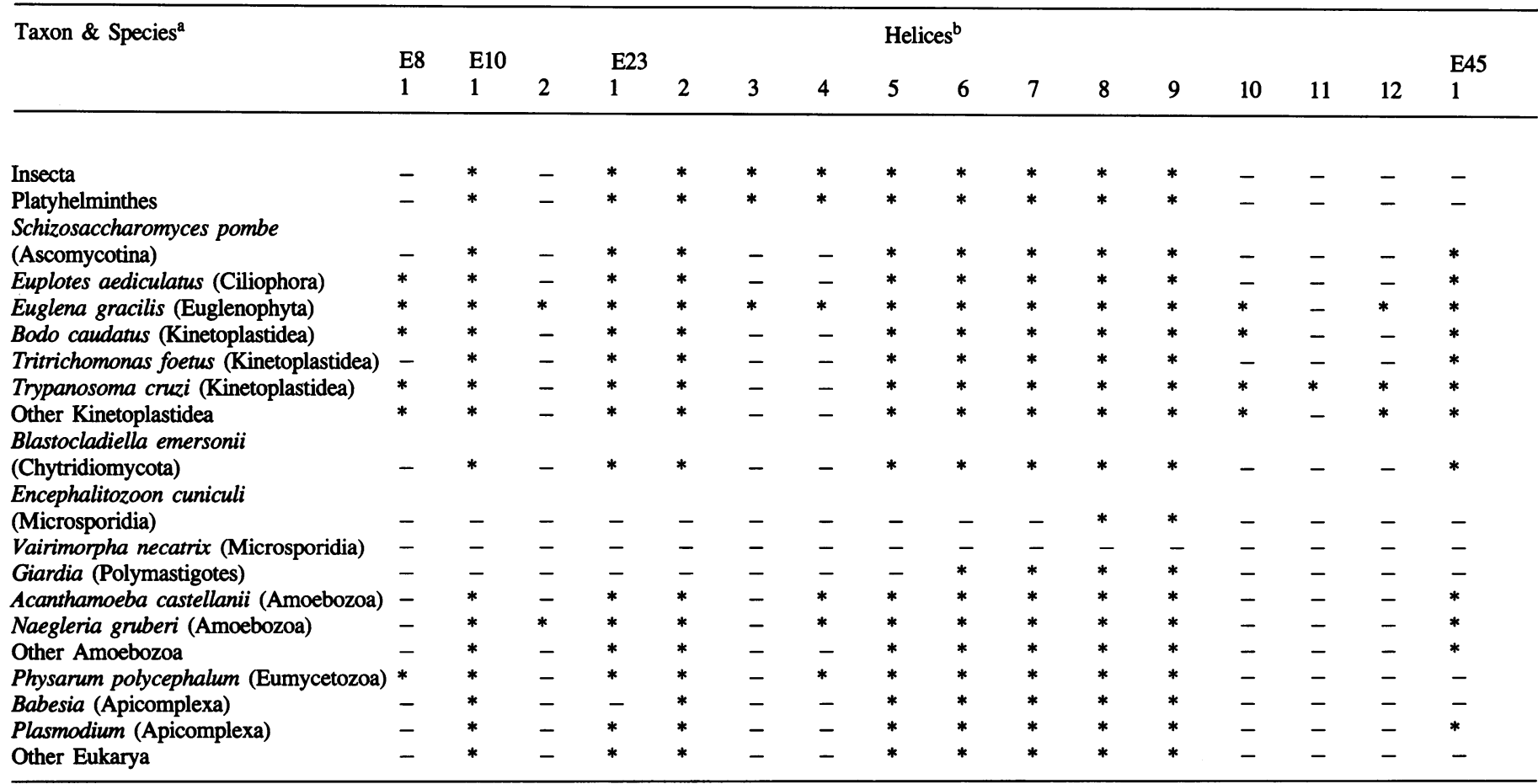

(a)In the case of the genera Giardia, Babesia, and Plasmodium, the helix occupancy applies to all species of the genus.

(b)The presence of a helix is indicated by an asterisk. Only eukaryote-specific helices are listed since universal helices are present in all known eukaryotic SSU rRNAs except those of Vairimorpha necatrix, which lacks helices 10,11, 43, and 46 and of Encephalitozoon cuniculi, which lacks helices 11, 18, 43, and 46. The structure of the SSU rRNA of the insect Acyrtosyphon pisum in area V4 (helices E23-n), which is exceptionally large in this species, is not yet known. 


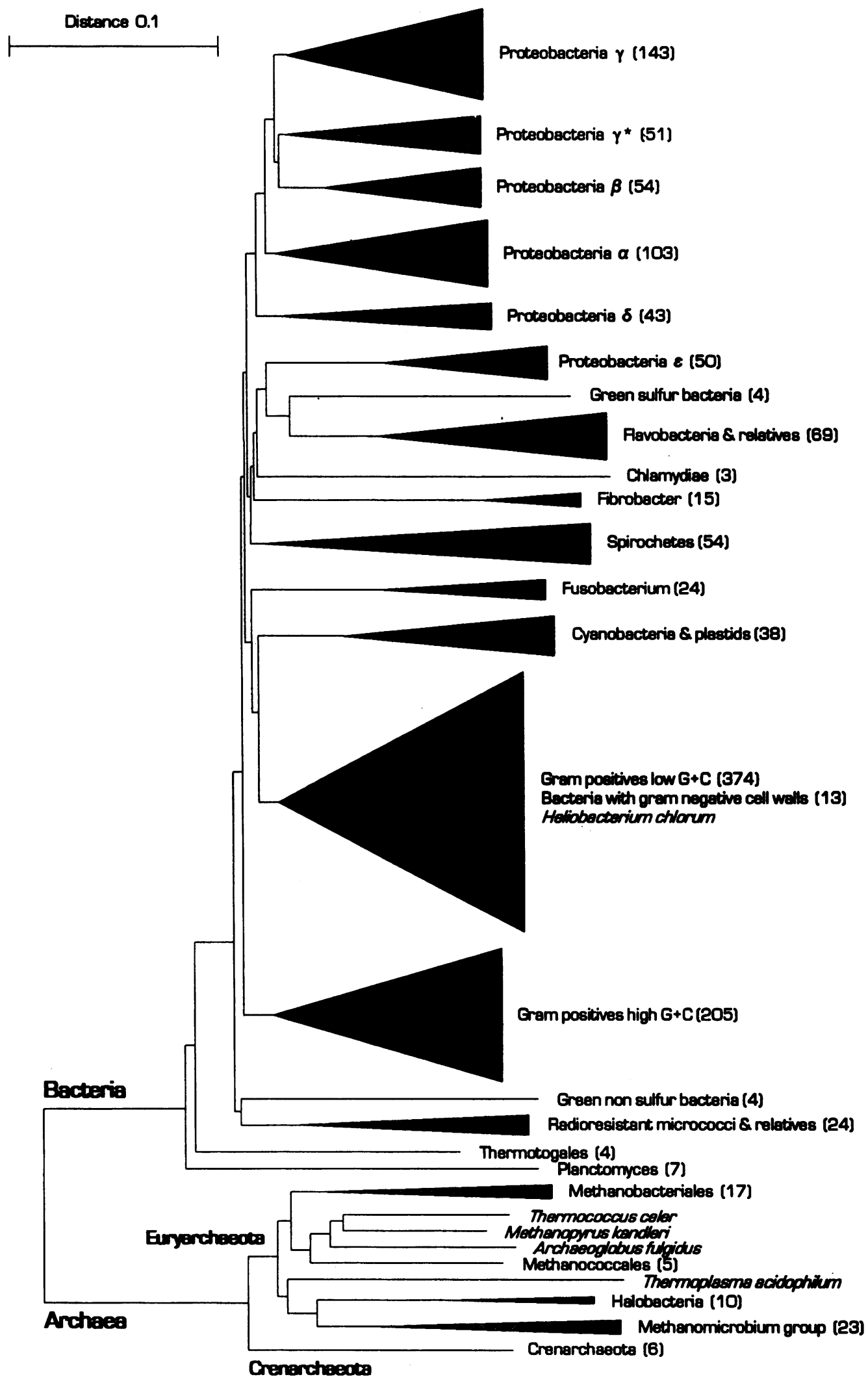

Figure 1. Evolutionary tree reconstructed from archaeal, bacterial and plastidial SSU rRNA sequences. The tree was constructed as described in the text from a total of 1348 SSU rRNA sequences. Clusters distinguishable in the tree are simplified to isosceles triangles with a height approximately equal to the average distance separating the terminal nodes from the deepest branching point within the cluster, and a base proportional to the number of sequences composing it, mentioned in brackets after the taxon name. If a taxon is represented by a single sequence, the species name is mentioned in italics. Each cluster corresponds to a taxon listed in Table 1. The cluster labeled Proteobacteria $\gamma^{*}$ is in fact more closely related to the Proteobacteria $\beta$ than to the Proteobacteria $\gamma$ in the present tree. However, its position between the latter two clusters is not stable and it consists mainly of species classified as Proteobacteria $\gamma$ by Woese (11) (see also footnote b to Table 1). The scale on top measures evolutionary distance expressed in substitutions per nucleotide. 


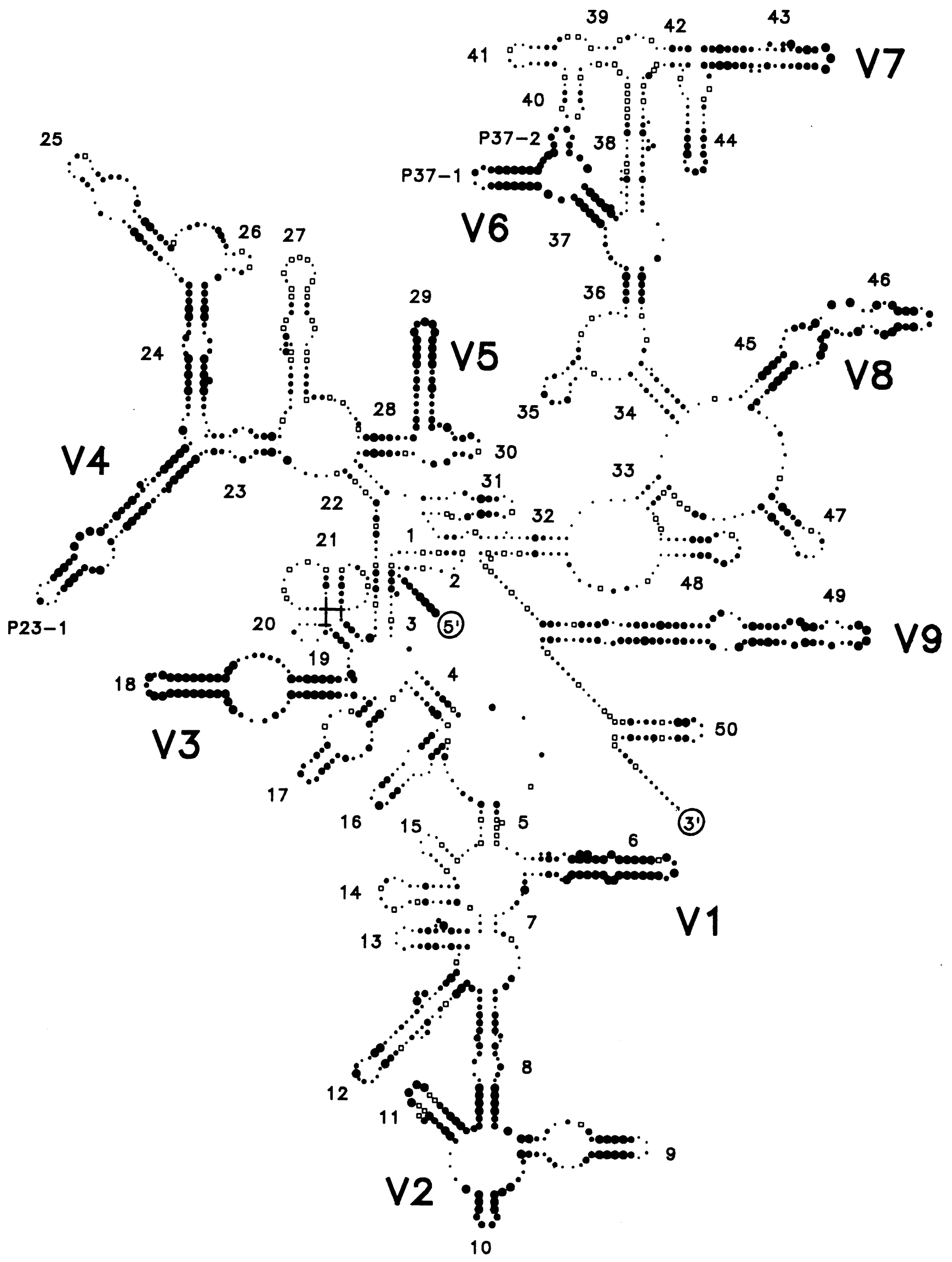

Figure 2. Secondary structure model for prokaryotic SSU rRNAs. Sites are divided into five equally numerous categories of increasing variability, indicated by full circles of increasing diameter. Sites that are invariant among presently known sequences are indicated as hollow squares. Areas containing the most variable sites are labeled V1 to V9. The helix numbering system is explained in the text. Helices P37-1 and P37-2 are absent in archaeal SSU rRNAs. 
3044 Nucleic Acids Research, 1993, Vol. 21, No. 13

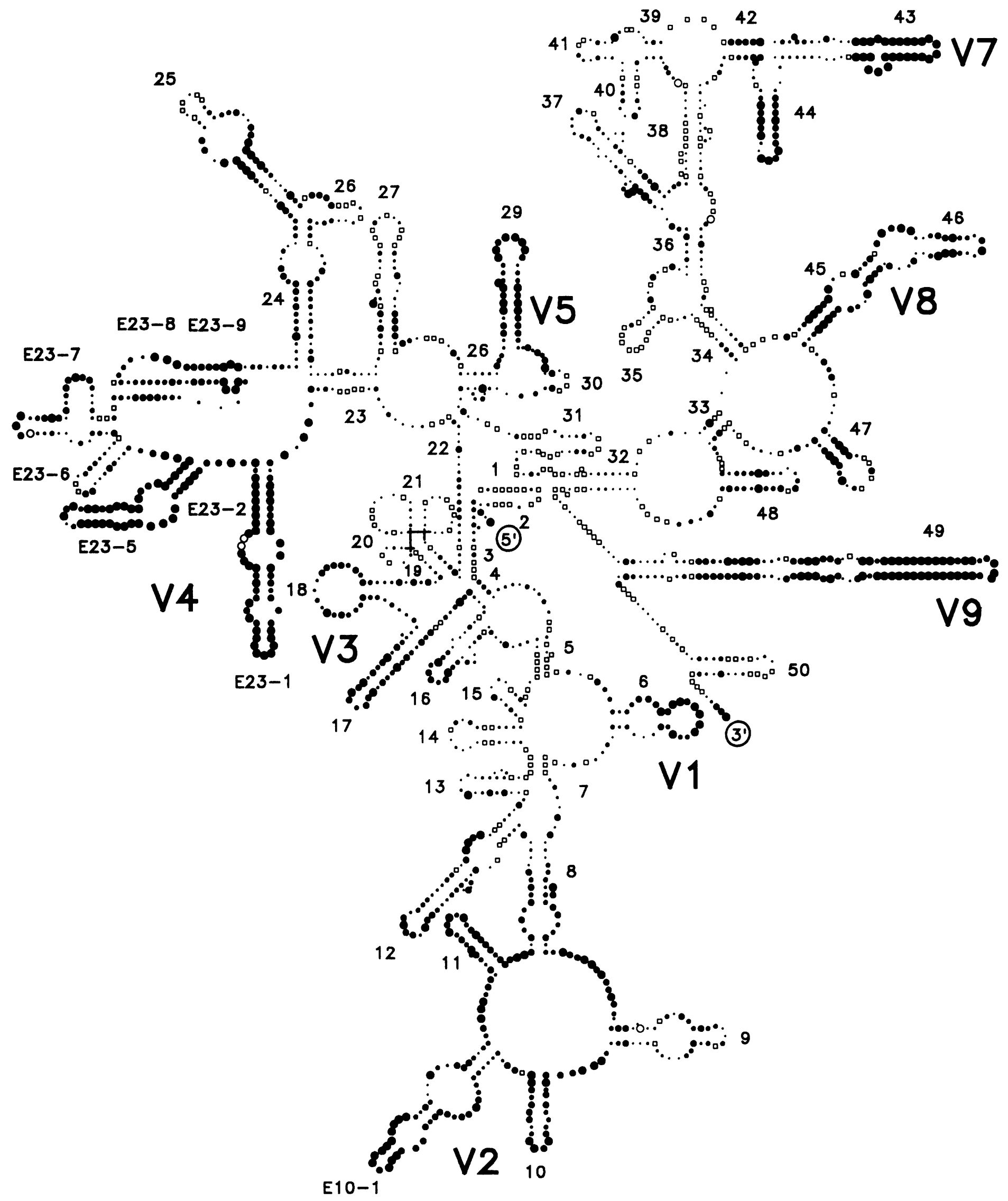

Figure 3. Secondary structure model for eukaryotic SSU rRNAs. Conventions are as in Fig. 2. The shape of the model is based on Saccharomyces cerevisiae SSU rRNA, and hollow circles represent nucleotides deleted in most other eukaryotic SSU rRNAs. The area corresponding to V6 in prokaryotic SSU rRNAs is more conserved among eukaryotic SSU rRNAs. 
Nucleic Acids Research, 1993, Vol. 21, No. 133045

Escherichia coli

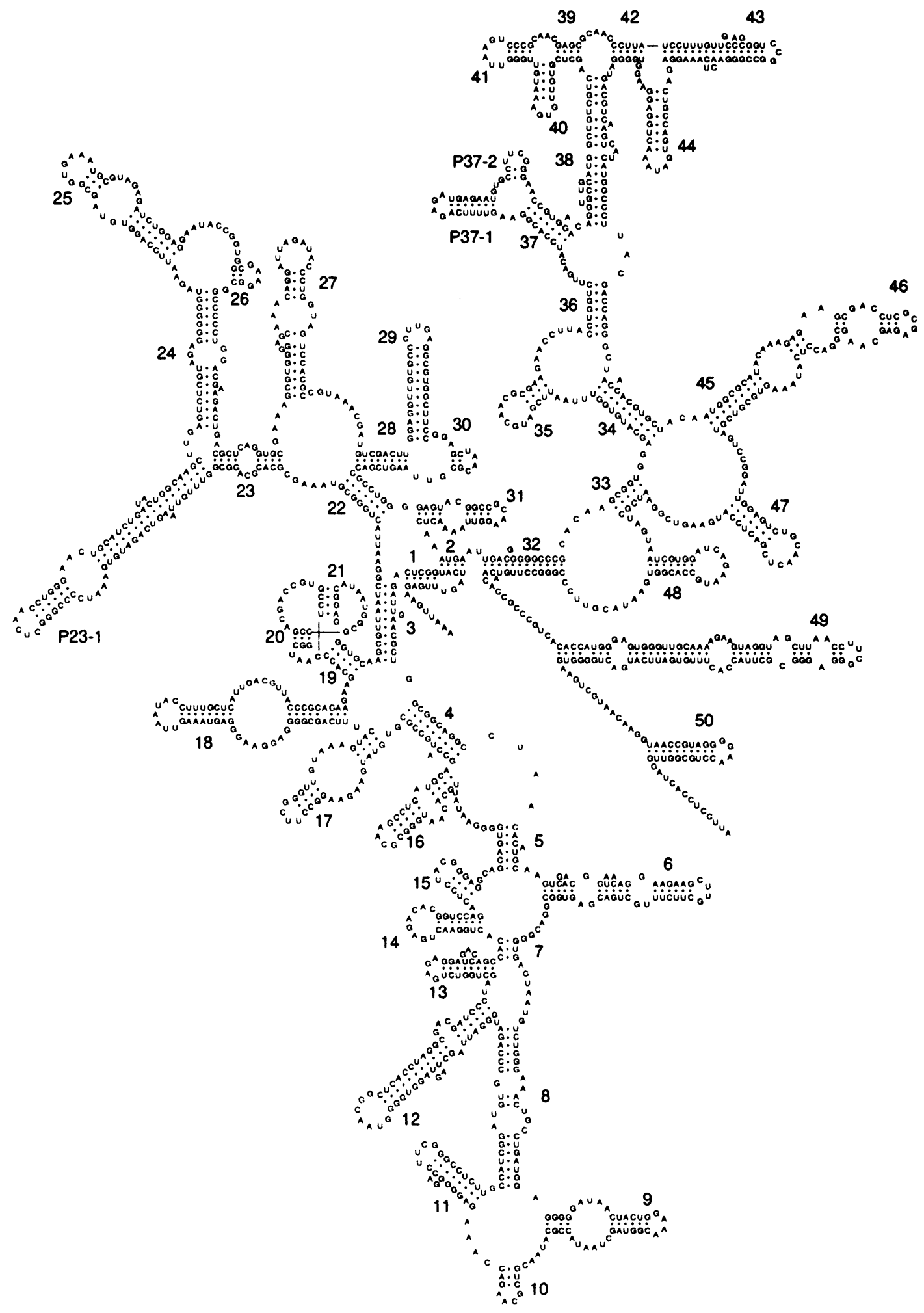

Figure 4. Secondary structure model for SSU rRNA of the bacterium Escherichia coli. 


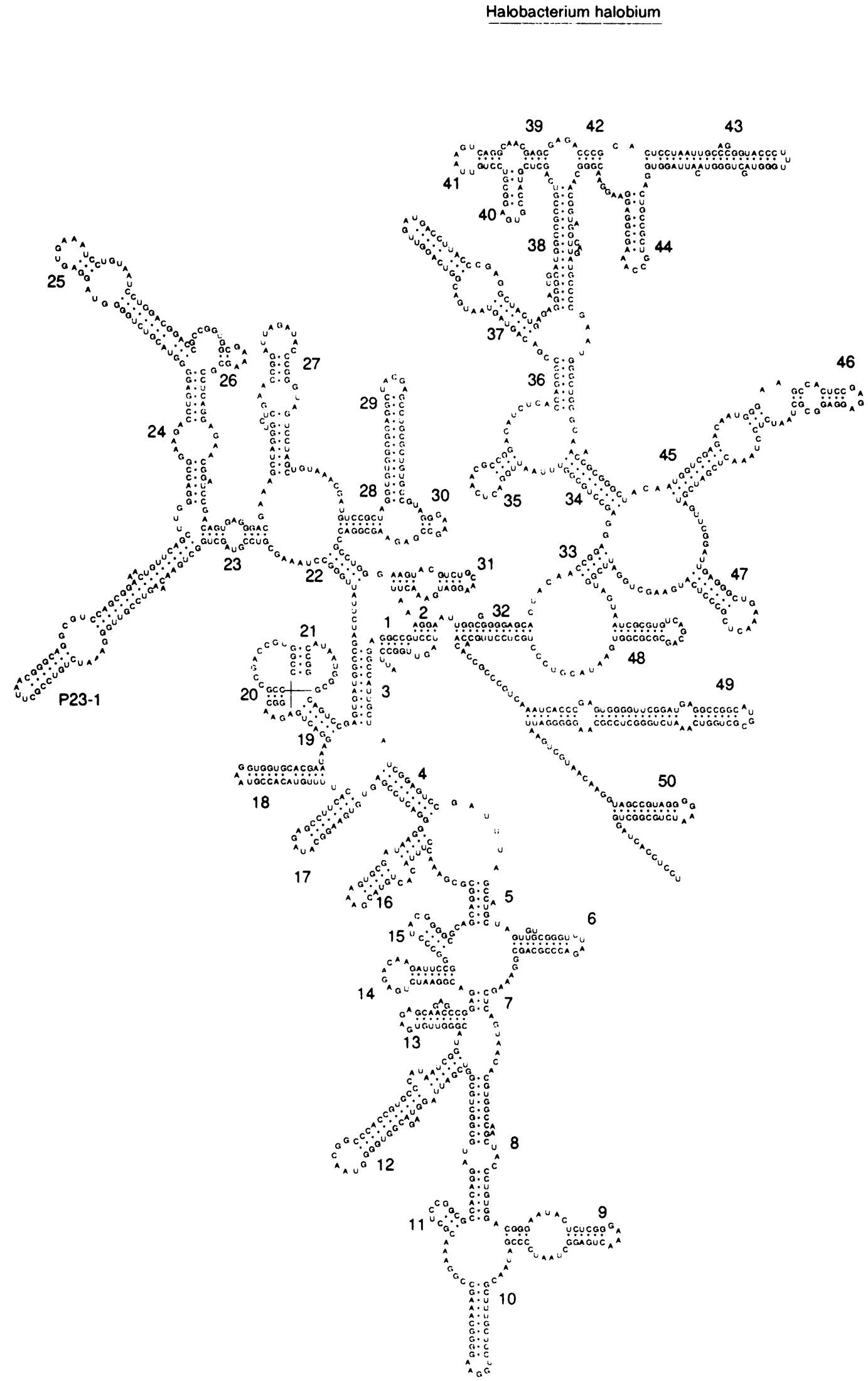

Figure 5. Secondary structure model for SSU rRNA of the archaebacterium Halobacterium halobium. 


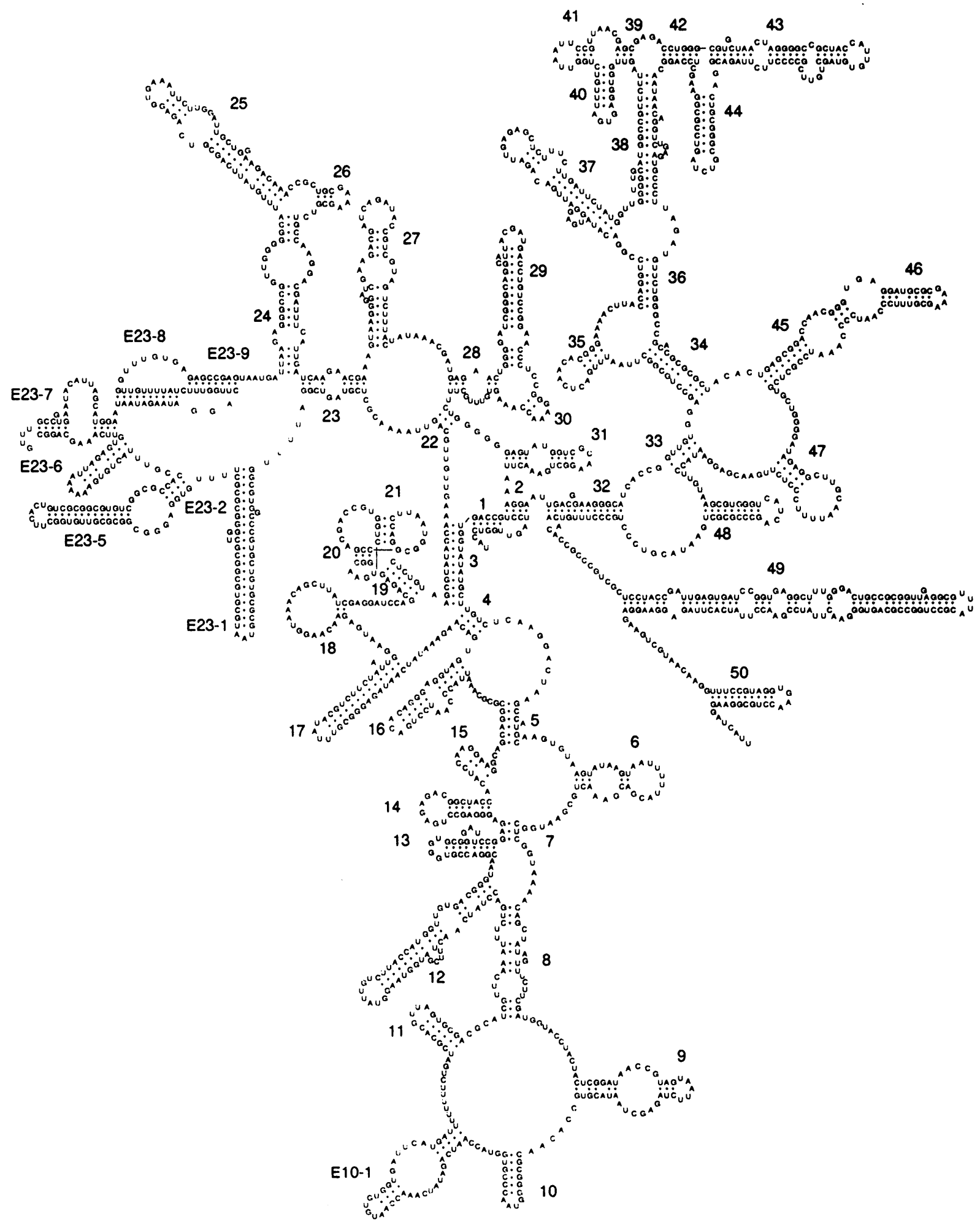

Figure 6. Secondary structure model for SSU rRNA of the red alga Palmaria palmata. 
3048 Nucleic Acids Research, 1993, Vol. 21, No. 13

Giardia duodenalis

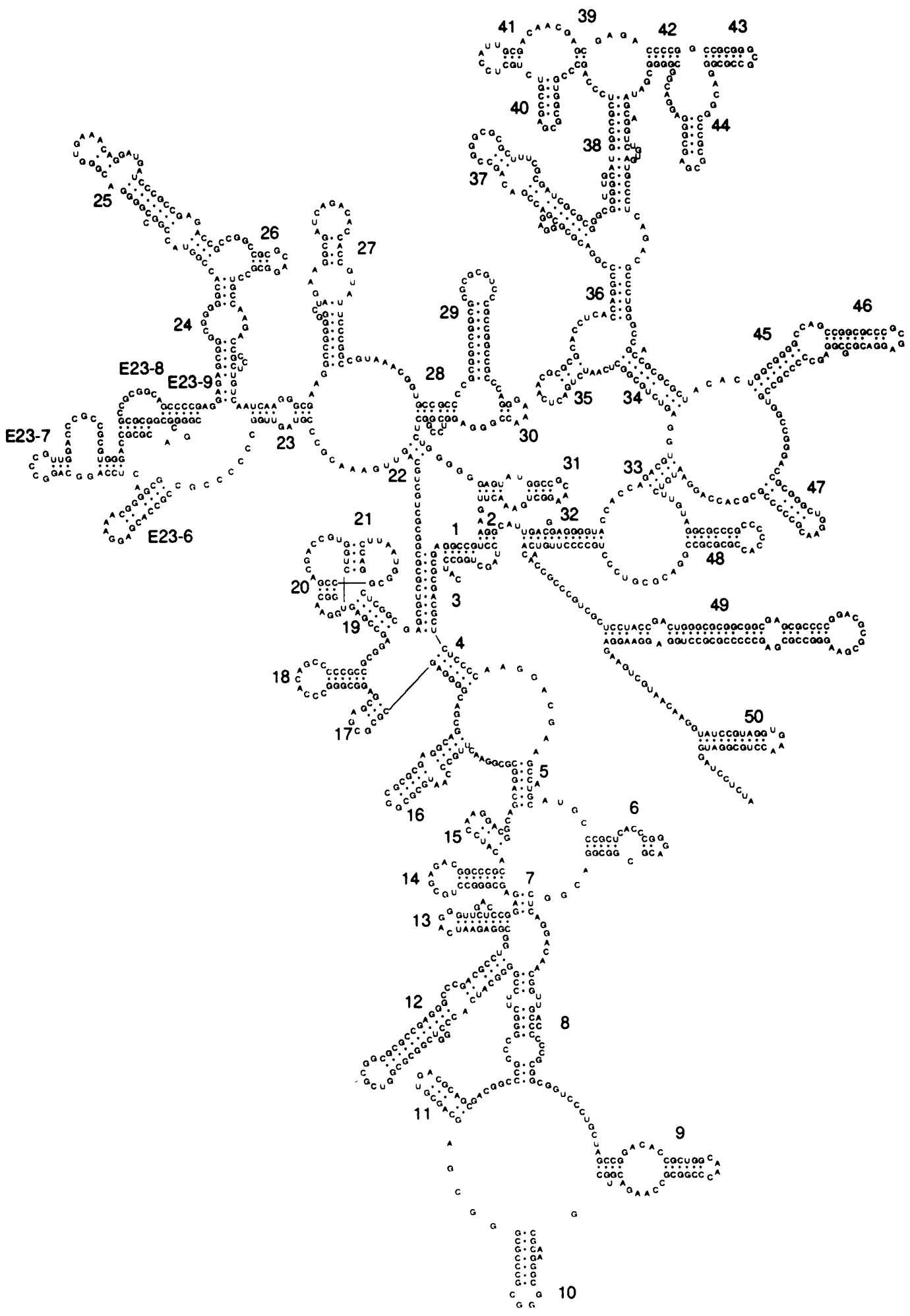

Figure 7. Secondary structure model for SSU rRNA of the polymastigote Giardia duodenalis. 


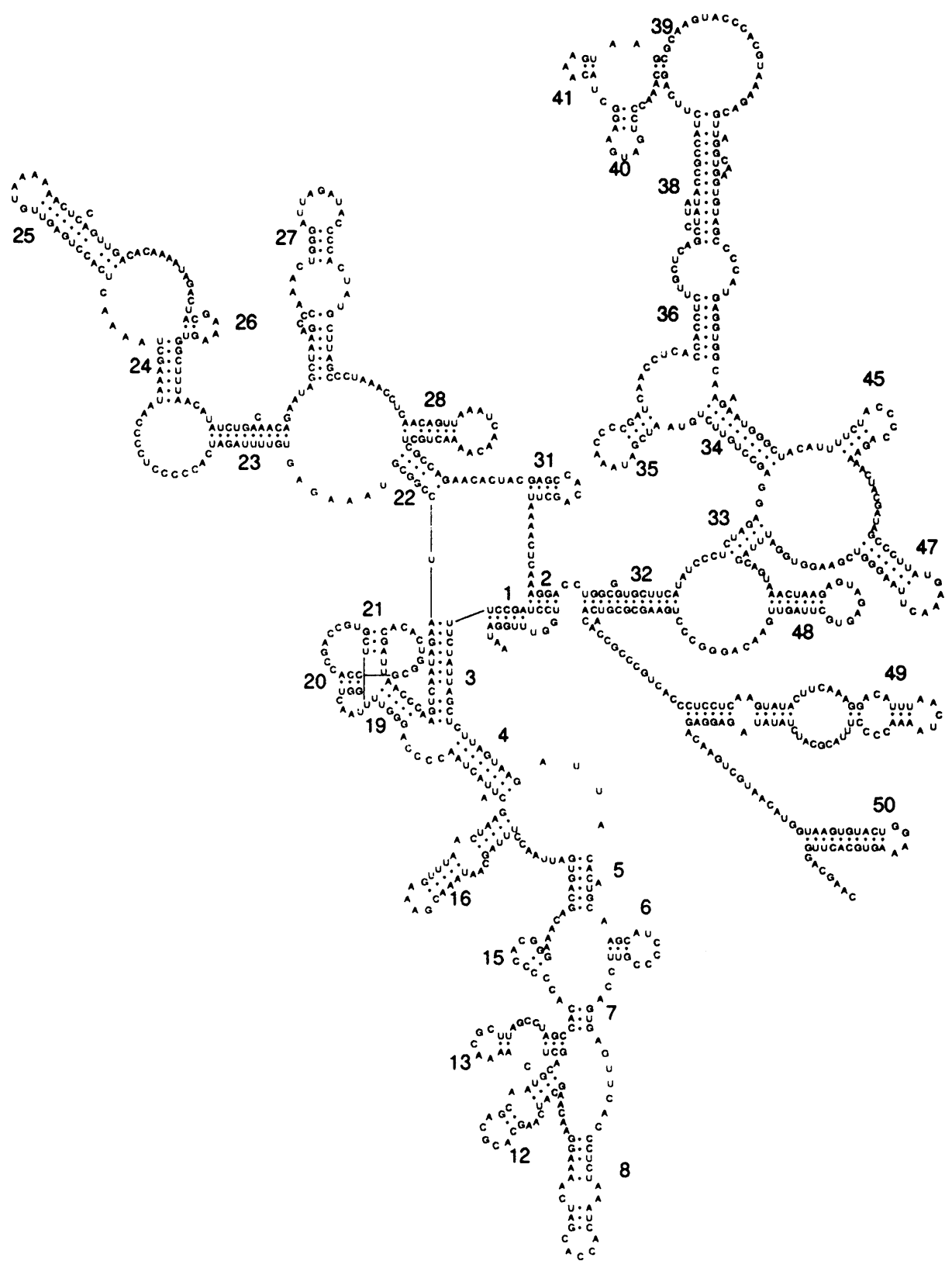

Figure 8. Secondary structure model for SSU rRNA of Homo sapiens mitochondrion. 